KEK-TH-1202

December 29, 2007

\title{
Unparticle physics at the photon collider
}

\author{
Tatsuru Kikuchi ${ }^{a}$ 1, Nobuchika Okada ${ }^{a, b} 2$ and Michihisa Takeuchi ${ }^{a, b, c} 3$ \\ a Theory Division, KEK, Oho 1-1, Tsukuba, Ibaraki, 305-0801, Japan \\ ${ }^{b}$ Department of Particle and Nuclear Physics, The Graduate University for Advanced Studies, \\ Oho 1-1, Tsukuba, Ibaraki, 305-0801, Japan \\ c Yukawa Institute for Theoretical Physics, Kyoto University, Kyoto, 606-8502, Japan
}

\begin{abstract}
Recently, a conceptually new physics beyond the Standard Model (SM), unparticle, has been proposed, where a hidden conformal sector is coupled to the SM sector through higher dimensional operators. In this setup, we investigate unparticle physics at the photon collider, in particular, unparticle effects on the $\gamma \gamma \rightarrow \gamma \gamma$ process. Since this process occurs at loop level in the SM, the unparticle effects can be significant even if the cutoff scale is very high. In fact, we find that the unparticle effects cause sizable deviations from the SM results. The scaling dimension of the unparticle $d_{\mathcal{U}}$ reflects the dependence of the cross section on the final state photon invariant mass, so that precision measurements of this dependence may reveal the scaling dimension of the unparticle.
\end{abstract}

\footnotetext{
${ }^{1}$ E-mail : tatsuru@post.kek.jp

${ }^{2}$ E-mail : okadan@post.kek.jp

${ }^{3}$ E-mail: tmichihi@post.kek.jp
} 


\section{Introduction}

The Large Hadron Collider (LHC), which will start its operation within a year, is expected to probe a new hitherto unexplored domain of particles and forces beyond the standard model around $\mathrm{TeV}$ scale. Although the LHC has the considerable potential to detect some indication of new physics beyond the Standard Model (SM), the detailed study of its properties needs more precise measurements and such a work will be performed at the International Linear Collider (ILC). According to the ILC Reference Design Report [1, the ILC is determined to run with $\sqrt{s}=500 \mathrm{GeV}$ and the total luminosity required is $\mathcal{L}=500 \mathrm{fb}^{-1}$ within the first four years of operation and $\mathcal{L}=1000 \mathrm{fb}^{-1}$ during the first phase of operation with $\sqrt{s}=500 \mathrm{GeV}$. An $e^{+} e^{-}$collider is uniquely capable of operation at a series of energies near the threshold of a new physics process. This is an extremely powerful tool for precision measurements of particle masses and unambiguous particle spin determinations. Various ILC physics studies indicate that a $\sqrt{s}=500 \mathrm{GeV}$ collider can have a great impact on understanding a new physics around TeV scale. An energy upgrade up to $\sqrt{s} \sim 1 \mathrm{TeV}$ would open the door to even greater discoveries.

Another very unique feature of the ILC is that it can be transformed into $\gamma \gamma$ collisions with the photon beams generated by using the Compton backscattering of the initial electron and laser beams. In this case, the energy and luminosity of the photon beams would be the same order of magnitude of the original electron beams. Since the set of final states at a photon collider is much richer than that in the $e^{+} e^{-}$mode, the photon collider would open a wider window to probe new physics beyond the SM. In fact, it has been seen in several new physics models that photon collider is more powerful for searching models than the $e^{+} e^{-}$ linear collider.

The most comprehensive description of the photon collider available at present is in a part of the TESLA TDR [2]. Also, there are some useful reviews for the physics at the photon collider as an option of the ILC [3, 4]. Since the high energy photon beams are provided through Compton scatterings from the electron beams, the $\gamma \gamma$ luminosity is determined by the geometric luminosity of the original electron beams [5]. For the standard ILC beam parameters, the $\gamma \gamma$ luminosity is expected to be $L_{\gamma \gamma}=0.17 \times L_{e e}$ with the integrated luminosity of the incident $e^{+} e^{-}$collider $\left(L_{e e}\right)$. Considering that cross sections in $\gamma \gamma$ are larger than those in $e^{+} e^{-}$collisions by one order of magnitude, the number of events will be somewhat larger than in $e^{+} e^{-}$collisions.

A certain class of new physics models includes a scalar field which is singlet under the SM gauge group. Such a new particle can have a direct coupling with photons suppressed by a new physics scale in low energy effective theory. If the new physics scale is low enough, the particle can be produced at the photon collider, and thus the photon collider can be a powerful tool to probe such a class of new physics models. In particular, the process, $\gamma \gamma \rightarrow \gamma \gamma$, is interesting because in the SM, this process occurs only at loop level and the SM background for new physics search is expected to be small. 
As one of such models, in this paper, we consider a new physics recently proposed by Georgi [6], which is described in terms of 'unparticle'. The unparticle physics is originated from a theory having some conformal fixed points in low energy, and the interaction between this conformal hidden sector and the SM sector is described by the effective theory at low energy. A concrete example of unparticle staff was proposed by Banks-Zaks [7] many years ago, where providing a suitable number of massless fermions, theory reaches a non-trivial infrared fixed points and a conformal theory can be realized at a low energy. Various phenomenological considerations on the unparticle physics have recently been developed in the literature [8, 9] as well as some studies on the formal aspects of the unparticle physics [10]. There have also been studied on the astrophysical and cosmological applications of the unparticle physics [11, 12], especially in [12], even the possibility for the unparticle to be a dark matter has been proposed.

In this paper, we investigate the unparticle physics at the photon collider. We concentrate on the process, $\gamma \gamma \rightarrow \gamma \gamma$, and the unparticle effects on it. As mentioned above, there is no tree level contribution in the SM, and we find that the unparticle effects cause sizable deviations from the SM results even if the cutoff scale of the higher dimensional interaction is of order $10 \mathrm{TeV}$.

\section{Basics of unparticle physics}

We begin with a brief review of the basic structure of the unparticle physics. First we introduce a coupling between the new physics operator $\left(\mathcal{O}_{\mathrm{UV}}\right)$ with dimension $d_{\mathrm{UV}}$ and the Standard Model one $\left(\mathcal{O}_{\mathrm{SM}}\right)$ with dimension $n$ at some ultraviolet $(\mathrm{UV})$ scale as

$$
\mathcal{L}=\frac{c_{n}}{M^{d_{\mathrm{UV}}+n-4}} \mathcal{O}_{\mathrm{UV}} \mathcal{O}_{\mathrm{SM}}
$$

where $c_{n}$ is a dimension-less constant, and $M$ is the energy scale characterizing the new physics. This new physics sector is assumed to become conformal at an IR scale $\Lambda_{\mathcal{U}}$, and the operator $\mathcal{O}_{\text {UV }}$ flows to the unparticle operator $\mathcal{U}$ with dimension $d_{\mathcal{U}}$. In low energy effective theory, we have the operator of the form,

$$
\mathcal{L}=c_{n} \frac{\Lambda_{\mathcal{U}}^{d_{\mathrm{UV}}-d_{\mathcal{U}}}}{M^{d_{\mathrm{UV}}+n-4}} \mathcal{U} \mathcal{O}_{\mathrm{SM}} \equiv \frac{\lambda_{n}}{\Lambda^{d_{\mathcal{U}}+n-4}} \mathcal{U} \mathcal{O}_{\mathrm{SM}},
$$

where the dimension of the unparticle $\mathcal{U}$ has been matched by $\Lambda_{\mathcal{U}}$ which is induced by the dimensional transmutation, $\lambda_{n}$ is an order one coupling constant and $\Lambda$ is the (effective) cutoff scale of low energy effective theory. In this paper, we consider only the scalar unparticle.

It was found in Ref. [6] that, by exploiting scale invariance of the unparticle, the phase space for an unparticle operator with the scaling dimension $d_{\mathcal{U}}$ and momentum $p$ is the same 
as the phase space for $d_{\mathcal{U}}$ invisible massless particles,

$$
d \Phi_{\mathcal{U}}(p)=A_{d_{\mathcal{U}}} \theta\left(p^{0}\right) \theta\left(p^{2}\right)\left(p^{2}\right)^{d_{\mathcal{U}}-2} \frac{d^{4} p}{(2 \pi)^{4}},
$$

where

$$
A_{d_{\mathcal{U}}}=\frac{16 \pi^{\frac{5}{2}}}{(2 \pi)^{2 d_{\mathcal{U}}}} \frac{\Gamma\left(d_{\mathcal{U}}+\frac{1}{2}\right)}{\Gamma\left(d_{\mathcal{U}}-1\right) \Gamma\left(2 d_{\mathcal{U}}\right)} .
$$

Also, based on the argument on the scale invariance, the (scalar) propagator for the unparticle was suggested to be [8]:

$$
\mathcal{P}\left(P^{2}\right)=\left\{\begin{array}{l}
Z_{d_{\mathcal{U}}} \times\left|P^{2}\right|^{d_{\mathcal{U}}-2} \quad\left(P^{2}<0\right) \\
Z_{d_{\mathcal{U}}} \times e^{-i \pi d_{\mathcal{U}}}\left|P^{2}\right|^{d_{\mathcal{U}}-2} \quad\left(P^{2}>0\right)
\end{array}\right.
$$

where $Z_{d_{\mathcal{U}}} \equiv \frac{A_{d_{\mathcal{U}}}}{2 \sin \left(\pi d_{\mathcal{U}}\right)}$ with $Z_{d_{\mathcal{U}} \rightarrow 1} \rightarrow-1$. Interestingly, $d_{\mathcal{U}}$ is not necessarily integer, it can be any real number or even complex number. In this paper we consider the scaling dimension in the range, $1 \leq d_{\mathcal{U}}<2$, for simplicity.

For our study on the photon collider, we consider the interaction between the unparticle and photons of the form 4 :

$$
\mathcal{L}_{\text {int }}=\frac{\mathcal{U}}{\Lambda^{d_{\mathcal{U}}}} F_{\mu \nu} F^{\mu \nu}
$$

This interaction causes the process $\gamma \gamma \rightarrow \gamma \gamma$ mediated by the unparticle in the $s, t$ and $u$-channels at the tree level.

\section{Unparticle effects at the Photon Collider}

Now we consider the effects of unparticle on the $\gamma \gamma \rightarrow \gamma \gamma$ process at the photon colliders. The helicity amplitude for the process

$$
\gamma\left(p_{1}, \lambda_{1}\right) \gamma\left(p_{2}, \lambda_{2}\right) \rightarrow \gamma\left(p_{3}, \lambda_{3}\right) \gamma\left(p_{4}, \lambda_{4}\right),
$$

is denoted as $\mathcal{M}_{\lambda_{1} \lambda_{2} \lambda_{3} \lambda_{4}}(\hat{s}, \hat{t}, \hat{u})$ 5, where $\hat{s}=\left(p_{1}+p_{2}\right)^{2}, \hat{t}=\left(p_{3}-p_{1}\right)^{2}, \hat{u}=\left(p_{4}-p_{1}\right)^{2}$. The Bose-Einstein statistics demands

$$
\begin{aligned}
& \mathcal{M}_{\lambda_{1} \lambda_{2} \lambda_{3} \lambda_{4}}(\hat{s}, \hat{t}, \hat{u})=\mathcal{M}_{\lambda_{2} \lambda_{1} \lambda_{4} \lambda_{3}}(\hat{s}, \hat{t}, \hat{u}) \\
& \mathcal{M}_{\lambda_{1} \lambda_{2} \lambda_{3} \lambda_{4}}(\hat{s}, \hat{t}, \hat{u})=\mathcal{M}_{\lambda_{2} \lambda_{1} \lambda_{3} \lambda_{4}}(\hat{s}, \hat{u}, \hat{t})
\end{aligned}
$$

\footnotetext{
${ }^{4}$ When we introduce all those kinds of terms between the unparticle and the SM gauge bosons, the process $g g \rightarrow \mathcal{U} \rightarrow \gamma \gamma$ has an impact on physics at hadron colliders such as the LHC and Tevatron. In particular, there is an impact on Higgs boson $(h)$ search through the gluon fusion process, $g g \rightarrow h \rightarrow \gamma \gamma$. Although such a process is out of our scope in this paper, it is worth investigating.

${ }^{5}$ We will use the notation for the matrix elements for the photon photon scattering amplitude as $\left\langle\gamma\left(p_{3}, \lambda_{3}\right) \gamma\left(p_{4}, \lambda_{4}\right) \mid \gamma\left(p_{1}, \lambda_{1}\right) \gamma\left(p_{2}, \lambda_{2}\right)\right\rangle=1+i(2 \pi)^{4} \delta^{4}\left(p_{1}+p_{2}-p_{3}-p_{4}\right) \mathcal{M}_{\lambda_{1} \lambda_{2} \lambda_{3} \lambda_{4}}$.
} 
while crossing symmetry implies

$$
\begin{aligned}
& \mathcal{M}_{\lambda_{1} \lambda_{2} \lambda_{3} \lambda_{4}}(\hat{s}, \hat{t}, \hat{u})=\mathcal{M}_{-\lambda_{4} \lambda_{2} \lambda_{3}-\lambda_{1}}(\hat{t}, \hat{s}, \hat{u})=\mathcal{M}_{\lambda_{1}-\lambda_{3}-\lambda_{2} \lambda_{4}}(\hat{t}, \hat{s}, \hat{u}) \\
& \mathcal{M}_{\lambda_{1} \lambda_{2} \lambda_{3} \lambda_{4}}(\hat{s}, \hat{t}, \hat{u})=\mathcal{M}_{-\lambda_{3} \lambda_{2}-\lambda_{1} \lambda_{4}}(\hat{u}, \hat{t}, \hat{s})=\mathcal{M}_{\lambda_{1}-\lambda_{4} \lambda_{3}-\lambda_{2}}(\hat{u}, \hat{t}, \hat{s})
\end{aligned}
$$

When parity and time inversion invariance holds, we have, respectively, the constraints

$$
\begin{aligned}
& \mathcal{M}_{\lambda_{1} \lambda_{2} \lambda_{3} \lambda_{4}}(\hat{s}, \hat{t}, \hat{u})=\mathcal{M}_{-\lambda_{1}-\lambda_{2}-\lambda_{3}-\lambda_{4}}(\hat{s}, \hat{t}, \hat{u}) \\
& \mathcal{M}_{\lambda_{3} \lambda_{4} \lambda_{1} \lambda_{2}}(\hat{s}, \hat{t}, \hat{u})=\mathcal{M}_{\lambda_{1} \lambda_{2} \lambda_{3} \lambda_{4}}(\hat{s}, \hat{t}, \hat{u}) .
\end{aligned}
$$

As a result, the 16 possible helicity amplitudes can be expressed in terms of only three independent amplitudes, $\mathcal{M}_{++++}(\hat{s}, \hat{t}, \hat{u}), \mathcal{M}_{+++-}(\hat{s}, \hat{t}, \hat{u})$ and $\mathcal{M}_{++--}(\hat{s}, \hat{t}, \hat{u})$, through the relations [15],

$$
\begin{aligned}
& \mathcal{M}_{ \pm \pm \mp \pm}(\hat{s}, \hat{t}, \hat{u})=\mathcal{M}_{ \pm \mp \pm \pm}(\hat{s}, \hat{t}, \hat{u})=\mathcal{M}_{ \pm \mp \mp \mp}(\hat{s}, \hat{t}, \hat{u})=\mathcal{M}_{ \pm \pm \pm \mp}(\hat{s}, \hat{t}, \hat{u}) \\
& \mathcal{M}_{--++}(\hat{s}, \hat{t}, \hat{u})=\mathcal{M}_{++--}(\hat{s}, \hat{t}, \hat{u}) \\
& \mathcal{M}_{ \pm \mp \pm \mp}(\hat{s}, \hat{t}, \hat{u})=\mathcal{M}_{----}(\hat{u}, \hat{t}, \hat{s})=\mathcal{M}_{++++}(\hat{u}, \hat{t}, \hat{s}), \\
& \mathcal{M}_{ \pm \mp \mp \pm}(\hat{s}, \hat{t}, \hat{u})=\mathcal{M}_{ \pm \mp \pm \mp}(\hat{s}, \hat{u}, \hat{t})=\mathcal{M}_{++++}(\hat{t}, \hat{s}, \hat{u})=\mathcal{M}_{++++}(\hat{t}, \hat{u}, \hat{s}) .
\end{aligned}
$$

Hence all the combinations can be expressed in terms of only three quantities, $\mathcal{M}_{++++}$, $\mathcal{M}_{++--}$and $\mathcal{M}_{+++-}$.

The resultant helicity amplitudes in the SM, $\mathcal{M}_{\lambda_{1} \lambda_{2} \lambda_{3} \lambda_{4}}^{\mathrm{SM}}$, are well-studied, for example, in [15, 16]. In the numerical calculation, we make use of LoopTools [17] for evaluating the loop functions that appear in the SM background.

It is easy to calculate the helicity amplitudes for the $\gamma \gamma \rightarrow \gamma \gamma$ process mediated by the unparticle in the $s, t$ and $u$-channels:

1. s-channel

$$
i \mathcal{M}_{\lambda_{1} \lambda_{2} \lambda_{3} \lambda_{4}}^{\mathcal{U}(s)}=-\frac{4 \hat{s}^{2}}{\Lambda^{2 d_{\mathcal{U}}}} \mathcal{P}(\hat{s}) \delta_{\lambda_{1}, \lambda_{2}} \delta_{\lambda_{3}, \lambda_{4}}
$$

2. t-channel

$$
i \mathcal{M}_{\lambda_{1} \lambda_{2} \lambda_{3} \lambda_{4}}^{\mathcal{U}(t)}=-\frac{4 \hat{t}^{2}}{\Lambda^{2 d_{\mathcal{U}}}} \mathcal{P}(\hat{t}) \delta_{\lambda_{1},-\lambda_{3}} \delta_{\lambda_{2},-\lambda_{4}}
$$

3. u-channel

$$
i \mathcal{M}_{\lambda_{1} \lambda_{2} \lambda_{3} \lambda_{4}}^{\mathcal{U}(u)}=-\frac{4 \hat{u}^{2}}{\Lambda^{2 d_{\mathcal{U}}}} \mathcal{P}(\hat{u}) \delta_{\lambda_{1},-\lambda_{3}} \delta_{\lambda_{2},-\lambda_{4}}
$$


The differential polarized cross section with respect to the scattering angle $\theta$ is given by

$$
\frac{d \hat{\sigma}^{\left(\lambda_{1} \lambda_{2}\right)}}{d \cos \theta}=\frac{1}{32 \pi \hat{s}} \sum_{\lambda_{3}, \lambda_{4}}\left[\left|\mathcal{M}_{\lambda_{1} \lambda_{2} \lambda_{3} \lambda_{4}}^{\mathrm{SM}}+\mathcal{M}_{\lambda_{1} \lambda_{2} \lambda_{3} \lambda_{4}}^{\mathcal{U}(s)}+\mathcal{M}_{\lambda_{1} \lambda_{2} \lambda_{3} \lambda_{4}}^{\mathcal{U}\left(t_{4}\right.}+\mathcal{M}_{\lambda_{1} \lambda_{2} \lambda_{3} \lambda_{4}}^{\mathcal{U}(u)}\right|^{2}\right] .
$$

The resultant cross sections in the limit $d_{\mathcal{U}} \rightarrow 1$, as a function of the photon beam energy are shown in Fig. 1. Here we have taken the cutoff scale to be $\Lambda=5 \mathrm{TeV}$. Contributions of the unparticle mediated processes become dominant as the beam energy becomes larger, as expected. The angular distribution of the cross section for $d_{\mathcal{U}} \rightarrow 1$ with a fixed photon beam energy, $\sqrt{\hat{s}}=500 \mathrm{GeV}$, is depicted in Fig. 2. The SM cross sections have a peak in the forward (and backward) region, while the cross sections of the unparticle mediated processes are almost flat, reflecting the 0 -spin of the scalar unparticle. Fig. 3 shows the resultant cross section as a function of the scaling dimension $d_{\mathcal{U}}$, for a fixed photon beam energy $\sqrt{\hat{s}}=500 \mathrm{GeV}$ and the cutoff scale $\Lambda=5 \mathrm{TeV}$. The unparticle effects quickly go down as $d_{\mathcal{U}}$ becomes larger, as expected in the results of the helicity amplitudes for the unparticle mediated processes.

In order to obtain the realistic cross section $\sigma(\gamma \gamma \rightarrow \gamma \gamma)$ at the photon collider, we convolute the fundamental cross section $\hat{\sigma}(\gamma \gamma \rightarrow \gamma \gamma)$ with the photon luminosity function. Throughout this paper, $\sqrt{s}$ refers to the center-of-mass energy of the incident $e^{+} e^{-}$collider and $\sqrt{\hat{s}}$ refers to the center of mass energy of the two incoming photons. The laser backscattering [2, 5] is the standard and efficient technique to convert an electron beam into a photon beam. The photon luminosity function $F_{\gamma / e}(x)$ is given by [2, 5]:

$$
F_{\gamma / e}\left(P_{e}, P_{\ell}, x\right)=\frac{1}{D(\xi)}\left[\frac{1}{1-x}-x+(2 r-1)^{2}-P_{e} P_{\ell} \xi r(2 r-1)(2-x)\right],
$$

where $P_{e}$ is the polarization of the initial electron and $P_{\ell}$ is the degree of circular polarization of the initial laser beam $\left(\left|P_{e}\right| \leq 1,\left|P_{\ell}\right| \leq 1\right), r=x / \xi(1-x)$, and $D(\xi)$ is a normalization factor,

$$
D(\xi)=\left(1-\frac{4}{\xi}-\frac{8}{\xi^{2}}\right) \ln (1+\xi)+\frac{1}{2}+\frac{8}{\xi}-\frac{1}{2(1+\xi)^{2}},
$$

with

$$
\xi=\frac{4 E_{0} \omega_{0}}{m_{e}^{2}}=4.475\left(\frac{2 E_{0}}{500 \mathrm{GeV}}\right)\left(\frac{\omega_{0}}{1.17 \mathrm{eV}}\right),
$$

where $E_{0}$ is the energy of the incident electron and $\omega_{0}$ is the energy of the incident laser photon. The Compton kinematics are characterized by the variable $x$, and one finds maximal energy conversion is given at $x_{\max }=\xi /(\xi+1)=0.817$ for $D(\xi=4.475) \simeq 1.77$. Then the maximum photon energy is given by $\omega_{\max }=x_{\max } E_{0} \simeq 0.82 E_{0}$. This means that about $82 \%$ of the incident electron-positron beam energy can be transmitted into the photon collider. One of the important observation is that the spectrum depends on the product of the helicity 
of the electron and the laser beam. The backscattered photons will retain a certain amount of the polarization of the laser photon beam. The hardest spectrum is provided by choosing the circular polarization of the laser $\left(P_{\ell}\right)$ and the mean helicity of the electron $\left(P_{e}\right)$ to be opposite, $P_{e} P_{\ell}=-1$, for both arms of the collider. $F_{\gamma / e}(0,0, x)$ corresponds to the unpolarized case.

Photon beam helicity is given by [2, 5]:

$$
\left\langle h_{\gamma}(x)\right\rangle \equiv \frac{-P_{\ell}(2 r-1)[1 /(1-x)+1-x]+P_{e} \xi r\left[1+(1-x)(2 r-1)^{2}\right]}{D(\xi) F_{\gamma / e}\left(P_{e}, P_{\ell}, x\right)} .
$$

By using the photon beam helicity, the total photon luminosity function can be decomposed according to each helicity component as

$$
F_{\gamma / e}^{ \pm}\left(x, P_{e}, P_{\ell}\right) \equiv \frac{1 \pm\left\langle h_{\gamma}(x)\right\rangle}{2} F_{\gamma / e}\left(P_{e}, P_{\ell}, x\right)
$$

There is a relation between different sign choices of polarization vectors:

$$
F_{\gamma / e}^{\lambda}\left(x, P_{e}, P_{\ell}\right)=F_{\gamma / e}^{-\lambda}\left(x,-P_{e},-P_{\ell}\right) .
$$

Then, the cross section for the polarized photon beam can be obtained by integrating over all the energy distributions:

$$
\begin{aligned}
\sigma(\gamma \gamma & \rightarrow \gamma \gamma) \\
& =\sum_{\lambda_{1}, \lambda_{2}} \int_{x_{1 \min }}^{x_{\max }} \int_{x_{2 \min }}^{x_{\max }} F_{\gamma / e}^{\lambda_{1}}\left(x_{1}, P_{e}, P_{\ell}\right) F_{\gamma / e}^{\lambda_{2}}\left(x_{2}, P_{e}^{\prime}, P_{\ell}^{\prime}\right) \hat{\sigma}^{\left(\lambda_{1} \lambda_{2}\right)}\left(\gamma \gamma \rightarrow \gamma \gamma ; \hat{s}=x_{1} x_{2} s\right) d x_{1} d x_{2} \\
& =\sum_{\lambda_{1}, \lambda_{2}} \int_{\tau_{\min }}^{\tau_{\max }} \frac{d \mathcal{L}^{\left(\lambda_{1} \lambda_{2}\right)}}{d \tau}\left(\tau, P_{e}, P_{\ell}, P_{e}^{\prime}, P_{\ell}^{\prime}\right) \hat{\sigma}^{\left(\lambda_{1} \lambda_{2}\right)}(\gamma \gamma \rightarrow \gamma \gamma ; \hat{s}=\tau s) d \tau
\end{aligned}
$$

where the maximum value of $x$ is given by $x_{\max }=\xi /(1+\xi)=0.817$. In the second line in the above, we made a change of variable, the corresponding maximal value of $\tau$ is $\tau_{\max }=x_{\max }^{2}=0.668$. In our numerical evaluation of Eq. (28), we introduce an infrared cutoff $\tau_{\min }=0.04(\sqrt{s}>100 \mathrm{GeV})$ which is necessary to avoid the infrared divergence in the cross section that mainly comes from the fermion loop contributions.

The luminosity function in the above formula is defined as follows:

$$
\begin{aligned}
\frac{d \mathcal{L}^{\left(\lambda_{1} \lambda_{2}\right)}}{d \tau}\left(\tau, P_{e}, P_{\ell}, P_{e}^{\prime}, P_{\ell}^{\prime}\right) & \equiv \int_{x_{1 \min }}^{x_{\max }} \int_{x_{2 \min }}^{x_{\max }} F_{\gamma / e}^{\lambda_{1}}\left(x_{1}, P_{e}, P_{\ell}\right) F_{\gamma / e}^{\lambda_{2}}\left(x_{2}, P_{e}^{\prime}, P_{\ell}^{\prime}\right) \delta\left(\tau-x_{1} x_{2}\right) d x_{1} d x_{2} \\
& =\int_{-y_{\max }}^{y_{\max }} F_{\gamma / e}^{\lambda_{1}}\left(\sqrt{\tau} e^{y}, P_{e}, P_{\ell}\right) F_{\gamma / e}^{\lambda_{2}}\left(\sqrt{\tau} e^{-y}, P_{e}^{\prime}, P_{\ell}^{\prime}\right) d y
\end{aligned}
$$

where $y_{\max } \equiv \frac{1}{2} \log \frac{\tau_{\max }}{\tau}$. 
Based on the relation of Eq. (27) and the exchange symmetry between $F_{\gamma / e}^{\lambda_{1}}$ and $F_{\gamma / e}^{\lambda_{2}}$ in the definition of the luminosity function, Eq. (29), it holds the following symmetry property:

$$
\begin{aligned}
\mathcal{L}^{+-}\left(P_{e}, P_{\ell}, P_{e}^{\prime}, P_{\ell}^{\prime}\right) & =\mathcal{L}^{++}\left(P_{e}, P_{\ell},-P_{e}^{\prime},-P_{\ell}^{\prime}\right), \\
\mathcal{L}^{-+}\left(P_{e}, P_{\ell}, P_{e}^{\prime}, P_{\ell}^{\prime}\right) & =\mathcal{L}^{++}\left(-P_{e},-P_{\ell}, P_{e}^{\prime}, P_{\ell}^{\prime}\right) \\
\mathcal{L}^{--}\left(P_{e}, P_{\ell}, P_{e}^{\prime}, P_{\ell}^{\prime}\right) & =\mathcal{L}^{++}\left(-P_{e}^{\prime},-P_{\ell}^{\prime},-P_{e},-P_{\ell}\right) .
\end{aligned}
$$

Hence, without loss of generality, we can consider only $\mathcal{L}^{++}\left(P_{e}, P_{\ell}, P_{e}^{\prime}, P_{\ell}^{\prime}\right)$. (Here, we denote $\frac{d \mathcal{L}^{\lambda_{1} \lambda_{2}}}{d \tau}(\tau)$ as $\mathcal{L}^{\lambda_{1} \lambda_{2}}$, for simplicity.)

Corresponding to Fig. 1, Fig. 2 and Fig. 3, the results after the convolution are shown in Fig. 4, Fig. 5] and Fig. 6. In these figures, we have used the unpolarized luminosity function to show the results in case of unpolarized beam. Fig. 4 shows the total cross section $\left(30^{\circ}<\theta<150^{\circ}\right)$ as a function of the incident $e^{+} e^{-}$collider energy $\sqrt{s}$. Fig. 5 shows the angular distribution of the cross section. Fig. 6] shows the total cross section $\left(30^{\circ}<\theta<150^{\circ}\right)$ for a fixed photon beam energy $\sqrt{s}=500 \mathrm{GeV}$ as a function of the scaling dimension $d_{\mathcal{U}}$. We can see that in the case with $d_{\mathcal{U}} \simeq 1$, there are sizable deviations from the SM results for $\sqrt{s}=500 \mathrm{GeV}$, for example, even though the cutoff scale is very high $\Lambda=5 \mathrm{TeV}$.

In Fig. 7, we show the differential cross section as a function of the invariant mass of the final state photons, $d \sigma / d M_{\gamma \gamma}$, for $\sqrt{s}=500 \mathrm{GeV}$ and for various $d_{\mathcal{U}}$ values. Here, we show the contributions from each helicity components $\left(\lambda_{1} \lambda_{2}\right)=(++)$ and from $\left(\lambda_{1} \lambda_{2}\right)=(+-)$, separately. However, only the sum of all these contributions is observable, and we show the result in Fig. 8 (top). The deviation from the SM result becomes larger as the invariant mass is raised. After imposing a lower energy cut for the photon invariant mass $\left(M_{\gamma \gamma}^{\text {cut }}\right)$ and the integration with respect to the invariant mass, we obtain the cross section as a function of $M_{\gamma \gamma}^{\text {cut }}$. Fig. 8 (bottom) shows the ratio of the signal cross section to the SM one as a function of $M_{\gamma \gamma}^{\text {cut }}$ for $\sqrt{s}=500 \mathrm{GeV}, \Lambda=5 \mathrm{TeV}$ and for various $d_{\mathcal{U}}$. The ratio becomes enhanced for larger $M_{\gamma \gamma}^{\text {cut }}$. The resultant cross sections show different behaviors as a function of $M_{\gamma \gamma}^{\text {cut }}$, for different $d_{\mathcal{U}}$. Therefore we can determine $d_{\mathcal{U}}$ by precisely measuring the cross sections ratio as a function of $M_{\gamma \gamma}^{\text {cut }}$. The results for $\sqrt{s}=1 \mathrm{TeV}$ are also shown in Fig. 9 and Fig. 10,

The results using the polarized beams 6 are shown in Figs. 11 16. In these figures, it is shown for all combinations of polarizations in the beam. It can be seen from these figures that the effects of the initial beam polarizations can drastically change the behavior of the differential cross section $d \sigma / d M_{\gamma \gamma}$ as a function of the invariant mass of the final state photons. We can enhance the signal over background ratio to choose an appropriate initial beam polarizations. For example, when we choose $\left(P_{e}, P_{\ell}, P_{e}^{\prime}, P_{\ell}^{\prime}\right)=(+--+)$ or $(-++-)$, the differential cross section $d \sigma / d M_{\gamma \gamma}$ has a sharp peak at high energy as shown in Fig. 14, Using these options, we can extract the information on $d_{\mathcal{U}}$.

\footnotetext{
${ }^{6}$ In this paper, we assume the ideal case, $100 \%$ polarized beams.
} 


\section{Summary}

We have considered the unparticle physics at the photon collider, in particular, the unparticle effects on the $\gamma \gamma \rightarrow \gamma \gamma$ process. Since this process occurs at loop level in the SM, the unparticle effects can be significant even if the cutoff scale is very high. We have analyzed the cross section for the $\gamma \gamma \rightarrow \gamma \gamma$ process, including the unparticle mediated process, and found that even for $\Lambda=5 \mathrm{TeV}$, the unparticle effects cause the sizable deviations from the SM results with the incident $e^{+} e^{-}$collider energy at $\sqrt{s}=500 \mathrm{GeV}$. The dependence of the differential cross section of the final state photon invariant mass $d \sigma / d M_{\gamma \gamma}$ reflects the scaling dimension of the unparticle $d_{\mathcal{U}}$, therefore precision measurements of these dependences may reveal the scaling dimension of the unparticle. It has also been shown that the effects of the initial beam polarizations can drastically change the behaviors of the cross sections. We can enhance the signal over background ratio in some magnitude by choosing the initial beam polarizations appropriate.

\section{Acknowledgments}

The work of T.K. is supported by the Research Fellowship of the Japan Society for the Promotion of Science (\#1911329). The work of N.O. is supported in part by the Grant-inAid for Scientific Research from the Ministry of Education, Science and Culture of Japan (\#18740170). We would like to thank M.M. Nojiri and Y. Okada for their useful comments and discussions.

\section{Note Added}

After completing this work, we became aware of a very recent paper [25], in which the same subject on the unparticle effects at the photon collider was studied. There exists a difference between their result and ours. As pointed out in [26, the phase factor $e^{-i d_{U} \pi}$ associated with the $s$-channel unparticle propagator might not be taken care of properly in [25] while we treated it correctly and our result is consistent with the one in [26]. 


\section{References}

[1] J. Brau, Y. Okada and N. Walker, "ILC Reference Design Report Volume 1 - Executive Summary," arXiv:0712.1950 [physics.acc-ph]; A. Djouadi, J. Lykken, K. Monig, Y. Okada, M. J. Oreglia and S. Yamashita, "ILC Reference Design Report Volume 2: PHYSICS AT THE ILC," arXiv:0709.1893 [hep-ph]; N. Phinney, N. Toge and N. Walker, "LC Reference Design Report Volume 3 - Accelerator," arXiv:0712.2361 [physics.acc-ph]; T. Behnke, C. Damerell, J. Jaros and A. Myamoto, "ILC Reference Design Report Volume 4 - Detectors," arXiv:0712.2356 [physics.ins-det].

[2] B. Badelek et al. [ECFA/DESY Photon Collider Working Group], "TESLA Technical Design Report, Part VI, Chapter 1: Photon collider at TESLA," Int. J. Mod. Phys. A 19, 5097 (2004) arXiv:hep-ex/0108012.

[3] F. Bechtel et al., Nucl. Instrum. Meth. A 564, 243 (2006) arXiv:physics/0601204.

[4] V. I. Telnov, Acta Phys. Polon. B 37, 1049 (2006) arXiv:physics/0604108.

[5] I. F. Ginzburg, G. L. Kotkin, V. G. Serbo and V. I. Telnov, Nucl. Instrum. Meth. 205 (1983) 47; I. F. Ginzburg, G. L. Kotkin, S. L. Panfil, V. G. Serbo and V. I. Telnov, Nucl. Instrum. Meth. A 219, 5 (1984); V. I. Telnov, Nucl. Instrum. Meth. A 294, 72 (1990).

[6] H. Georgi, Phys. Rev. Lett. 98, 221601 (2007) arXiv:hep-ph/0703260.

[7] T. Banks and A. Zaks, Nucl. Phys. B 196, 189 (1982).

[8] H. Georgi, Phys. Lett. B 650, 275 (2007) [arXiv:0704.2457 [hep-ph]]; K. Cheung, W. Y. Keung and T. C. Yuan, Phys. Rev. Lett. 99, 051803 (2007) arXiv:0704.2588 [hep-ph]].

[9] M. Luo and G. Zhu, Phys. Lett. B 659, 341 (2008) arXiv:0704.3532 [hep-ph]]; C. H. Chen and C. Q. Geng, Phys. Rev. D 76, 115003 (2007) arXiv:0705.0689 [hepph]]; G. J. Ding and M. L. Yan, Phys. Rev. D 76, 075005 (2007) arXiv:0705.0794 [hepph]]; Y. Liao, Phys. Rev. D 76, 056006 (2007) [arXiv:0705.0837 [hep-ph]]; T. M. Aliev, A. S. Cornell and N. Gaur, Phys. Lett. B 657, 77 (2007) arXiv:0705.1326 [hep-ph]; X. Q. Li and Z. T. Wei, Phys. Lett. B 651, 380 (2007) arXiv:0705.1821 [hep-ph]; M. Duraisamy, arXiv:0705.2622 [hep-ph]; C. D. Lu, W. Wang and Y. M. Wang, Phys. Rev. D 76, 077701 (2007) arXiv:0705.2909 [hep-ph]]; P. J. Fox, A. Rajaraman and Y. Shirman, Phys. Rev. D 76, 075004 (2007) arXiv:0705.3092 [hep-ph]]; N. Greiner, Phys. Lett. B 653, 75 (2007) [arXiv:0705.3518 [hep-ph]]; D. Choudhury, D. K. Ghosh and Mamta, Phys. Lett. B 658, 148 (2008) arXiv:0705.3637 [hep-ph]]; S. L. Chen 
and X. G. He, Phys. Rev. D 76, 091702 (2007) arXiv:0705.3946 [hep-ph]]; T. M. Aliev, A. S. Cornell and N. Gaur, JHEP 0707, 072 (2007) arXiv:0705.4542 [hep-ph]]; P. Mathews and V. Ravindran, Phys. Lett. B 657, 198 (2007) arXiv:0705.4599 [hep-ph]]; S. Zhou, arXiv:0706.0302 [hep-ph]; G. J. Ding and M. L. Yan, arXiv:0706.0325 [hep-ph]; C. H. Chen and C. Q. Geng, Phys. Rev. D 76, 036007 (2007) [arXiv:0706.0850 [hep-ph]]; Y. Liao and J. Y. Liu, Phys. Rev. Lett. 99, 191804 (2007) [arXiv:0706.1284 [hep-ph]]; M. Bander, J. L. Feng, A. Rajaraman and Y. Shirman, Phys. Rev. D 76, 115002 (2007) arXiv:0706.2677 [hep-ph]]; T. G. Rizzo, JHEP 0710, 044 (2007) arXiv:0706.3025 [hep-ph]]; K. Cheung, W. Y. Keung and T. C. Yuan, Phys. Rev. D 76, 055003 (2007) arXiv:0706.3155 [hep-ph]]; H. Goldberg and P. Nath, arXiv:0706.3898 [hep-ph]; S. L. Chen, X. G. He and H. C. Tsai, JHEP 0711, 010 (2007) arXiv:0707.0187 [hepph]]; R. Zwicky, arXiv:0707.0677 [hep-ph]; T. Kikuchi and N. Okada, arXiv:0707.0893 [hep-ph]; R. Mohanta and A. K. Giri, Phys. Rev. D 76, 075015 (2007) arXiv:0707.1234 [hep-ph]]; C. S. Huang and X. H. Wu, arXiv:0707.1268 [hep-ph]; A. Lenz, Phys. Rev. D 76, 065006 (2007) arXiv:0707.1535 [hep-ph]]; J. J. van der Bij and S. Dilcher, Phys. Lett. B 655, 183 (2007) arXiv:0707.1817 [hep-ph]]; D. Choudhury and D. K. Ghosh, arXiv:0707.2074 [hep-ph]; H. Zhang, C. S. Li and Z. Li, Phys. Rev. D 76, 116003 (2007) arXiv:0707.2132 [hep-ph]]; X. Q. Li, Y. Liu and Z. T. Wei, arXiv:0707.2285 [hep-ph]; Y. Nakayama, Phys. Rev. D 76, 105009 (2007) arXiv:0707.2451 [hepph]]; N. G. Deshpande, X. G. He and J. Jiang, Phys. Lett. B 656, 91 (2007) arXiv:0707.2959 [hep-ph]]; R. Mohanta and A. K. Giri, Phys. Rev. D 76, 057701 (2007) arXiv:0707.3308 [hep-ph]]; A. Delgado, J. R. Espinosa and M. Quiros, JHEP 0710, 094 (2007) [arXiv:0707.4309 [hep-ph]]; G. Cacciapaglia, G. Marandella and J. Terning, arXiv:0708.0005 [hep-ph]; M. Neubert, arXiv:0708.0036 [hep-ph]; M. Luo, W. Wu and G. Zhu, Phys. Lett. B 659, 349 (2008) arXiv:0708.0671 [hep-ph]; N. G. Deshpande, S. D. H. Hsu and J. Jiang, arXiv:0708.2735 [hep-ph]; G. Bhattacharyya, D. Choudhury and D. K. Ghosh, Phys. Lett. B 655, 261 (2007) arXiv:0708.2835 [hep-ph]]; Y. Liao, arXiv:0708.3327 [hep-ph]; A. T. Alan and N. K. Pak, arXiv:0708.3802 [hepph]; I. Gogoladze, N. Okada and Q. Shafi, arXiv:0708.4405 [hep-ph]; C. H. Chen and C. Q. Geng, arXiv:0709.0235 [hep-ph]; T. i. Hur, P. Ko and X. H. Wu, Phys. Rev. D 76, 096008 (2007) arXiv:0709.0629 [hep-ph]]; L. Anchordoqui and H. Goldberg, arXiv:0709.0678 [hep-ph]; S. Majhi, arXiv:0709.1960 [hep-ph]; M. C. Kumar, P. Mathews, V. Ravindran and A. Tripathi, arXiv:0709.2478 [hep-ph]; S. Das, S. Mohanty and K. Rao, arXiv:0709.2583 [hep-ph]; G. j. Ding and M. L. Yan, arXiv:0709.3435 [hep-ph]; A. B. Balantekin and K. O. Ozansoy, Phys. Rev. D 76, 095014 (2007) arXiv:0710.0028 [hep-ph]]; T. M. Aliev and M. Savci, arXiv:0710.1505[hep-ph]; E. O. Iltan, arXiv:0710.2677 [hep-ph]; S. L. Chen, X. G. He, X. Q. Li, H. C. Tsai and Z. T. Wei, arXiv:0710.3663 [hep-ph]; A. T. Alan, N. K. Pak and A. Senol, arXiv:0710.4239 [hepph]; R. Zwicky, arXiv:0710.4430 [hep-ph]; O. Cakir and K. O. Ozansoy, arXiv:0710.5773 [hep-ph]; I. Sahin and B. Sahin, arXiv:0711.1665 [hep-ph]; E. O. Iltan, arXiv:0711.2744 
[hep-ph]; A. T. Alan, arXiv:0711.3272 [hep-ph]; K. Cheung, C. S. Li and T. C. Yuan, arXiv:0711.3361 [hep-ph]; R. Mohanta and A. K. Giri, arXiv:0711.3516 [hep-ph]; K. Huitu and S. K. Rai, arXiv:0711.4754 [hep-ph]; J. R. Mureika, arXiv:0712.1786 [hep-ph]; B. Holdom, arXiv:0712.2379 [hep-ph].

[10] M. A. Stephanov, Phys. Rev. D 76, 035008 (2007) arXiv:0705.3049 [hep-ph]]; N. V. Krasnikov, Int. J. Mod. Phys. A 22, 5117 (2007) [arXiv:0707.1419 [hep-ph]]; T. A. Ryttov and F. Sannino, Phys. Rev. D 76, 105004 (2007) arXiv:0707.3166 [hep-th]]; A. Kobakhidze, Phys. Rev. D 76, 097701 (2007) arXiv:0709.3782 [hep-ph]]; C. Germani and A. Schelpe, arXiv:0712.2243 [hep-th].

[11] H. Davoudiasl, Phys. Rev. Lett. 99, 141301 (2007) arXiv:0705.3636 [hep-ph]]; S. Hannestad, G. Raffelt and Y. Y. Y. Wong, Phys. Rev. D 76, 121701 (2007) arXiv:0708.1404 [hep-ph]]; P. K. Das, Phys. Rev. D 76, 123012 (2007) arXiv:0708.2812 [hep-ph]]; D. Majumdar, arXiv:0708.3485 [hep-ph]; A. Freitas and D. Wyler, JHEP 0712, 033 (2007) arXiv:0708.4339 [hep-ph]; J. McDonald, arXiv:0709.2350 [hep-ph]; S. L. Chen, X. G. He, X. P. Hu and Y. Liao, arXiv:0710.5129 [hep-ph]; S. Dutta and A. Goyal, arXiv:0712.0145 [hep-ph].

[12] T. Kikuchi and N. Okada, arXiv:0711.1506 [hep-ph].

[13] J. F. Gunion, H. E. Haber, G. L. Kane and S. Dawson, The Higgs Hunter's Guide, Addison-Wesley, Redwood City, California, 1989.

[14] S. Mrenna and J. Wells, Phys. Rev. D 63, 015006 (2000) arXiv:hep-ph/0001226.

[15] G. Jikia and A. Tkabladze, Phys. Lett. B 323, 453 (1994) arXiv:hep-ph/9312228].

[16] G. J. Gounaris, P. I. Porfyriadis and F. M. Renard, Eur. Phys. J. C 9, 673 (1999) [arXiv:hep-ph/9902230].

[17] T. Hahn and M. Perez-Victoria, Comput. Phys. Commun. 118, 153 (1999) arXiv:hep-ph/9807565]; see also, G. J. van Oldenborgh and J. A. M. Vermaseren, Z. Phys. C 46, 425 (1990).

[18] G. J. Gounaris, P. I. Porfyriadis and F. M. Renard, Phys. Lett. B 452, 76 (1999) [Erratum-ibid. B 513, 431 (2001)] |arXiv:hep-ph/9812378].

[19] E. Boos, A. De Roeck, I. F. Ginzburg, K. Hagiwara, R. D. Heuer, G. Jikia, J. Kwiecinski, D. J. Miller, T. Takahashi, V. Telnov, T. Rizzo, I. Watanabe, P. M. Zerwas, Nucl. Instrum. Meth. A 472, 100 (2001) arXiv:hep-ph/0103090|.

[20] B. Grzadkowski and J. F. Gunion, Phys. Lett. B 294, 361 (1992) arXiv:hep-ph/9206262]. 
[21] D. L. Borden, D. A. Bauer and D. O. Caldwell, Phys. Rev. D 48, 4018 (1993).

[22] J. F. Gunion and H. E. Haber, Phys. Rev. D 48, 5109 (1993).

[23] K. Hagiwara, S. Matsumoto, D. Haidt and C. S. Kim, Z. Phys. C 64, 559 (1994) [Erratum-ibid. C 68, 352 (1995)] arXiv:hep-ph/9409380].

[24] For a discussion to include the effect of a conformal symmetry breaking or the mass of the unparticle, which could be induced from the VEV of the Higgs field, especially, in scope of the photon collider physics, please refer, T. Kikuchi, N. Okada and M. Takeuchi, work in progress.

[25] O. Cakir and K. O. Ozansoy, arXiv:0712.3814v1 [hep-ph].

[26] C. F. Chang, K. Cheung and T. C. Yuan, arXiv:0801.2843 [hep-ph]. 


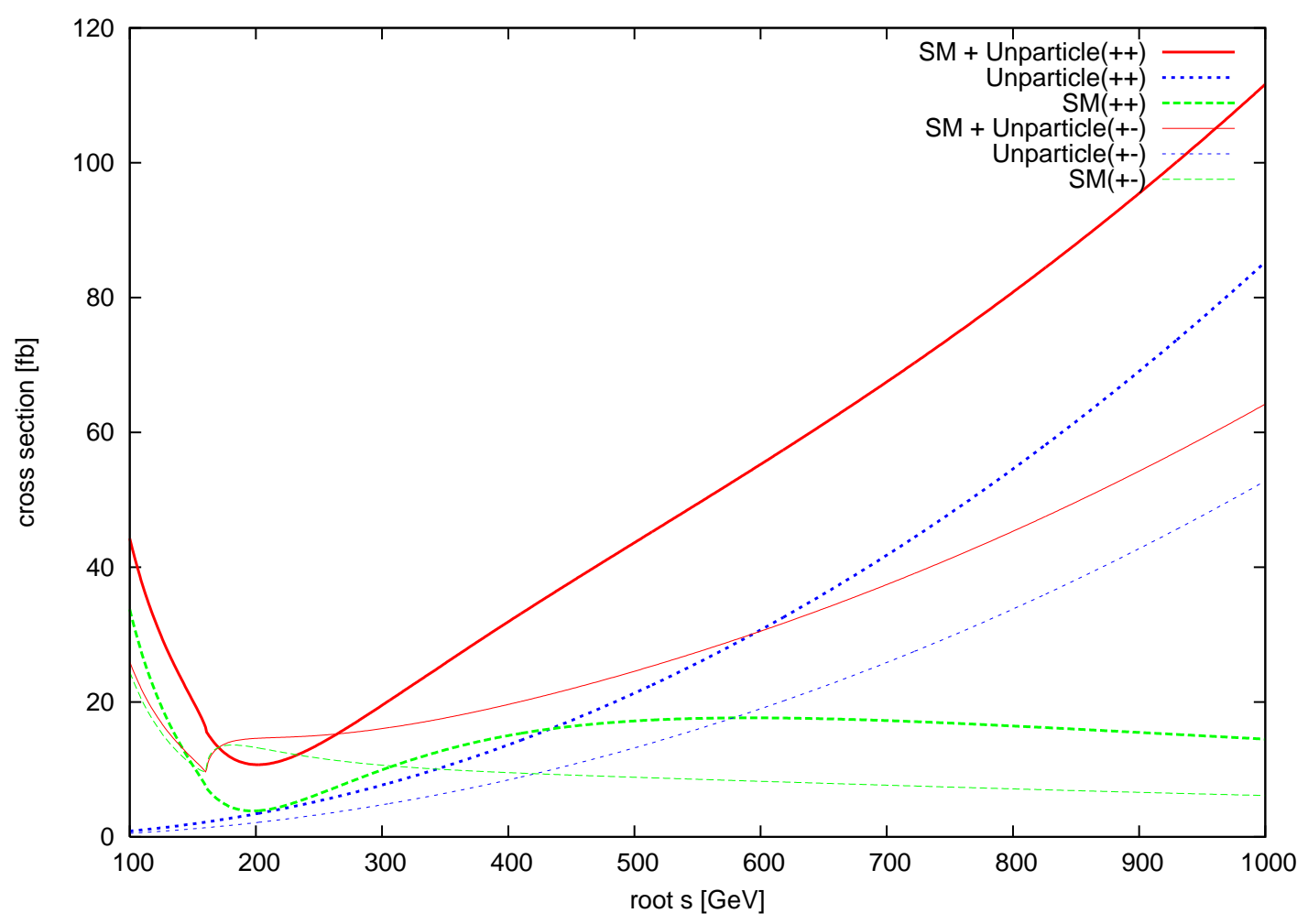

Figure 1: The total cross section of the scattering, $\gamma \gamma \rightarrow \gamma \gamma$, for the Standard Model process, purely unparticle contribution, and the combined result as a function of the photon energy $\sqrt{\hat{s}}$. Here we have taken the limit $d_{\mathcal{U}} \rightarrow 1$ and the cutoff scale to be $\Lambda=5 \mathrm{TeV}$. In the integration with respect to the scattering angle, we have imposed a cut as $30^{\circ}<\theta<150^{\circ}$. Two possible combinations of the initial photon helicities $\left(\lambda_{1} \lambda_{2}\right)=(++),(+-)$ are taken into account in this analysis, and the results are shown by different thickness of each lines. 


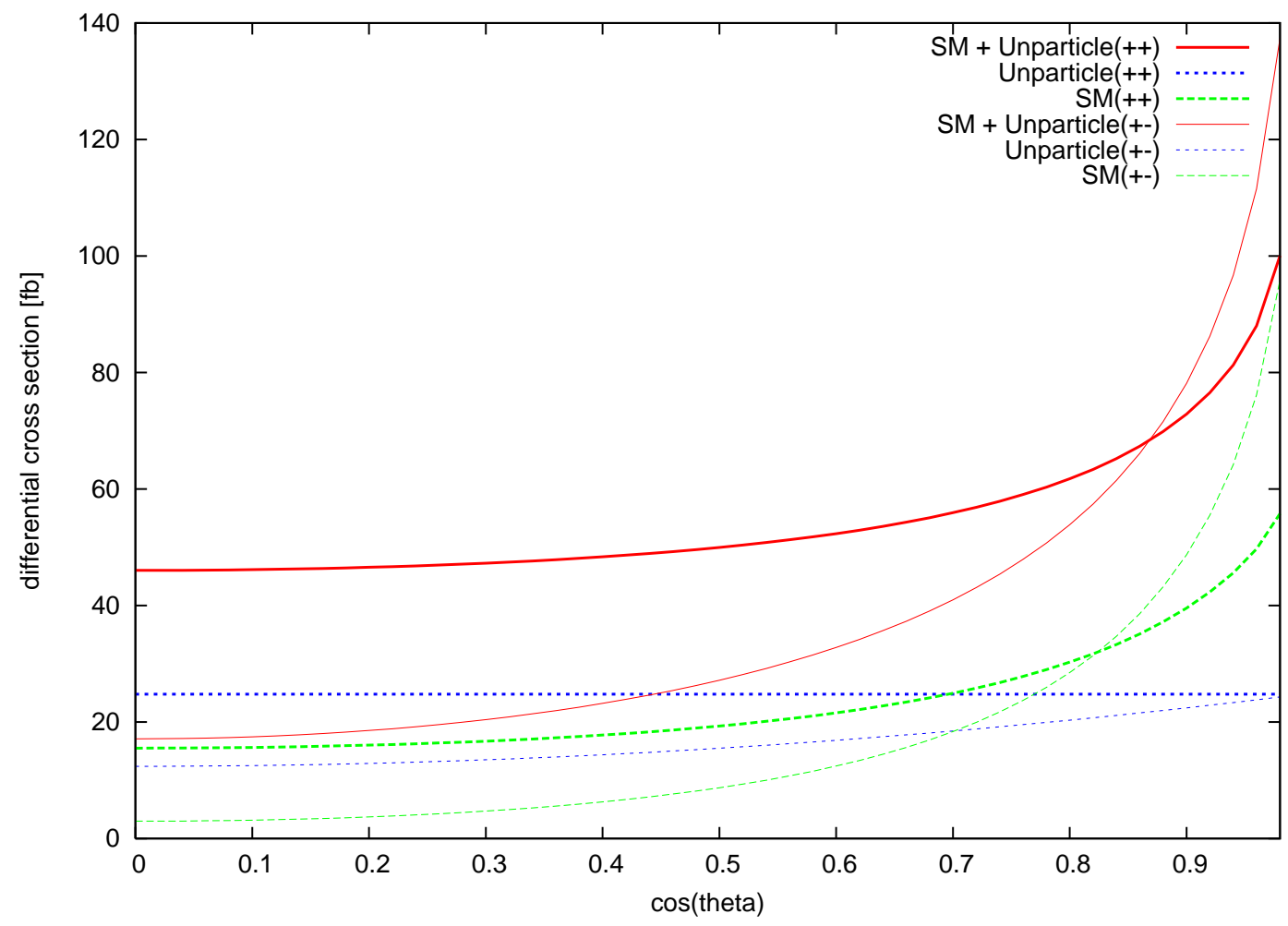

Figure 2: The angular distribution for the $\gamma \gamma \rightarrow \gamma \gamma$ process with the initial photon energy $\sqrt{\hat{s}}=500 \mathrm{GeV}$ and the cutoff scale $\Lambda=5 \mathrm{TeV}$, in the limit of $d_{\mathcal{U}} \rightarrow 1$. 


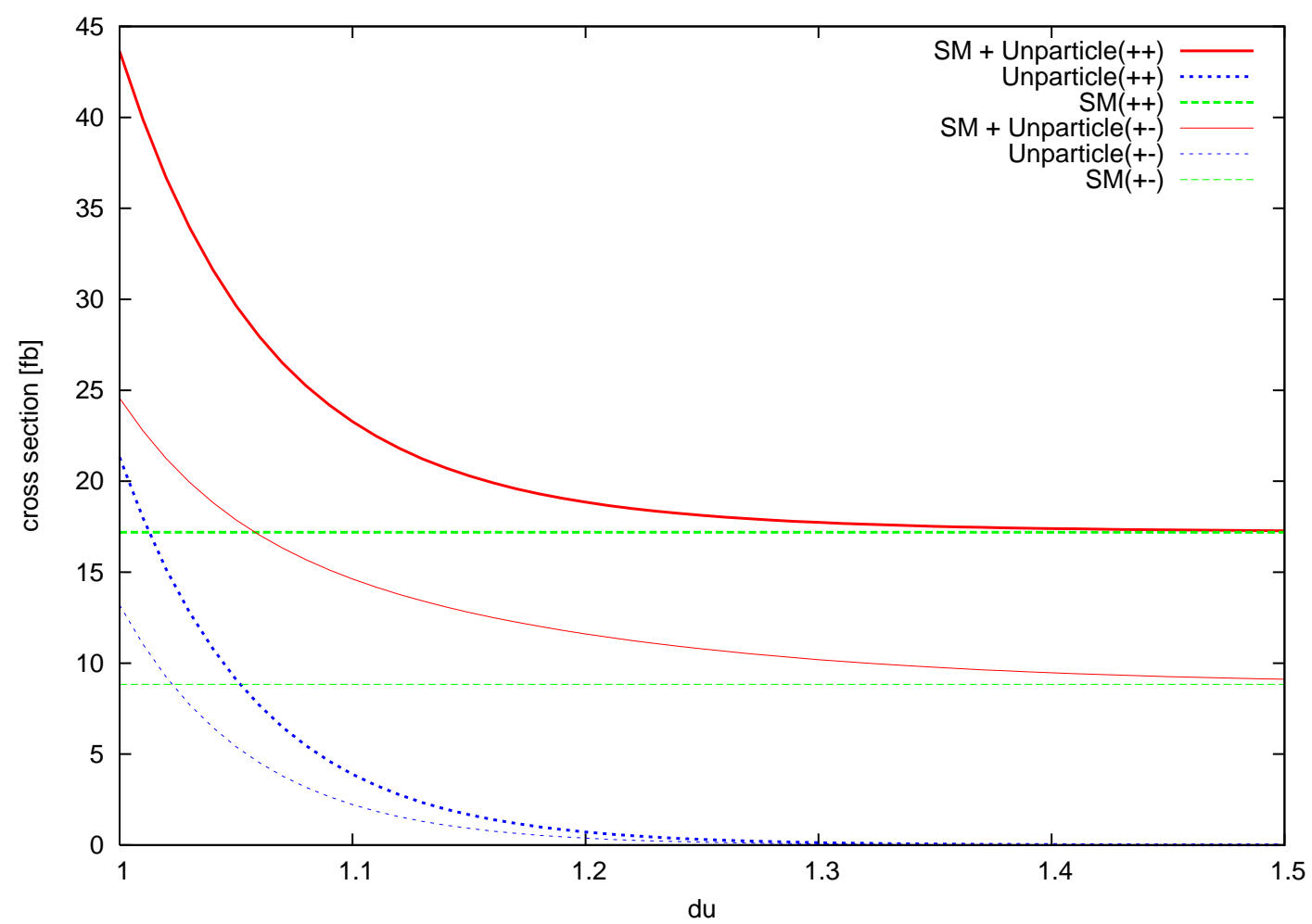

Figure 3: The total cross section of the $\gamma \gamma \rightarrow \gamma \gamma$ process for the Standard Model contribution, the pure unparticle contribution, and the combined result as a function of the scaling dimension, $d_{\mathcal{U}}$, for the initial photon energy $\sqrt{\hat{s}}=500 \mathrm{GeV}$ and $\Lambda=5 \mathrm{TeV}$. We have again imposed a cut for the scattering angle as $30^{\circ}<\theta<150^{\circ}$ in the integration. 


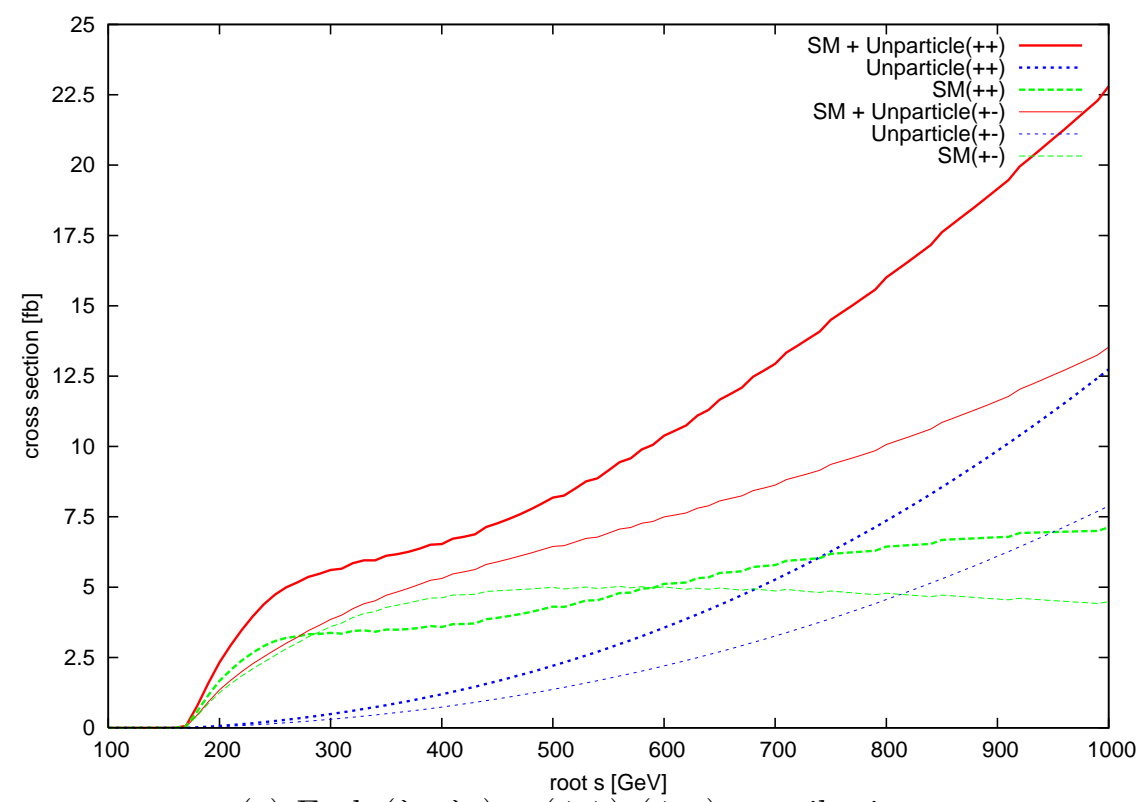

(a) Each $\left(\lambda_{1}, \lambda_{2}\right)=( \pm \pm),( \pm \mp)$ contribution

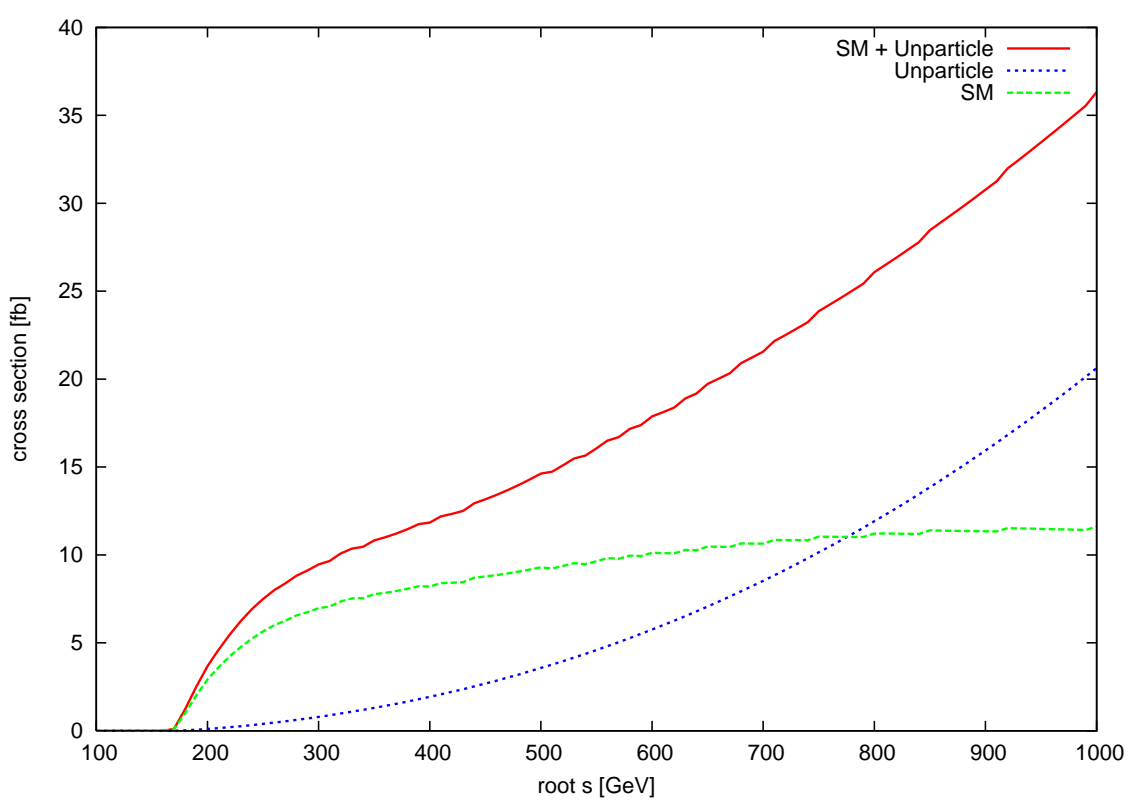

(b) Sum of both contributions

Figure 4: The convoluted cross section of the $\gamma \gamma \rightarrow \gamma \gamma$ process in the case of unpolarized beams. The figure shows for the Standard Model case, pure unparticle case and the combined result as a function of the incident $e^{+} e^{-}$collider energy $\sqrt{s}$. The top figure shows each contribution for $\left(\lambda_{1}, \lambda_{2}\right)=( \pm \pm)$ and $( \pm \mp)$. The bottom figure shows the sum of both contributions. 


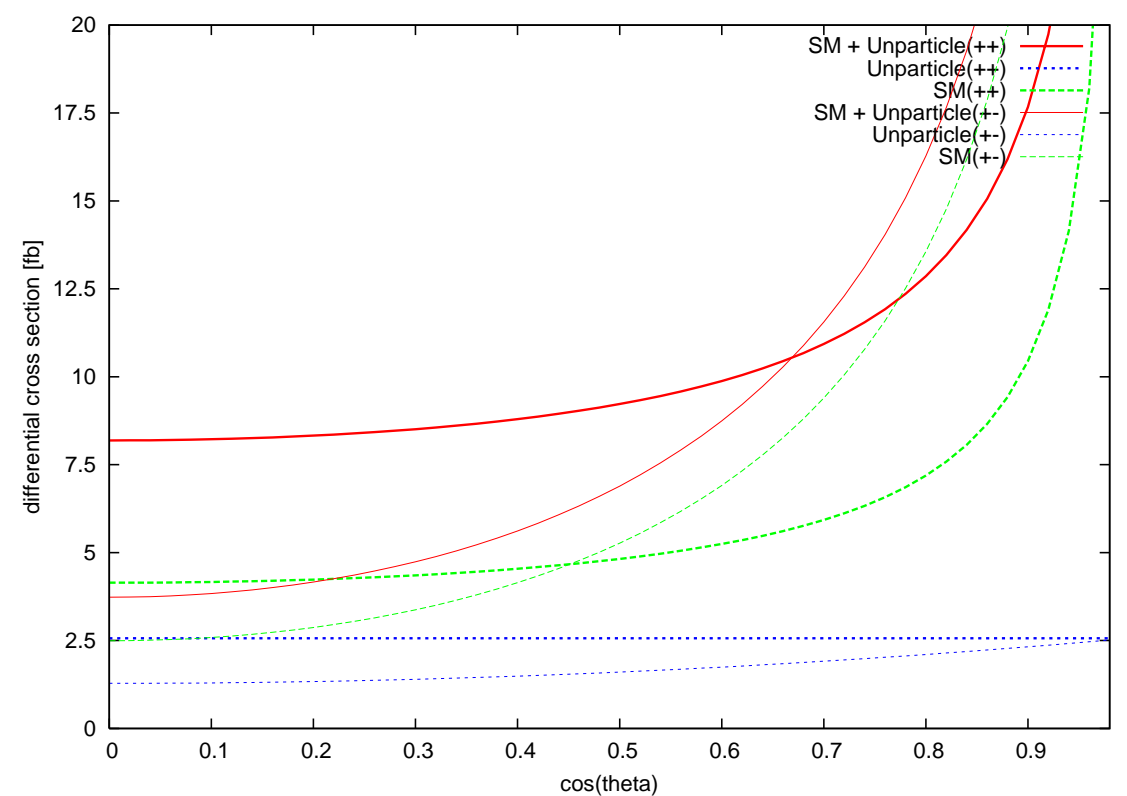

(a) Each $\left(\lambda_{1}, \lambda_{2}\right)=( \pm \pm),( \pm \mp)$ contribution

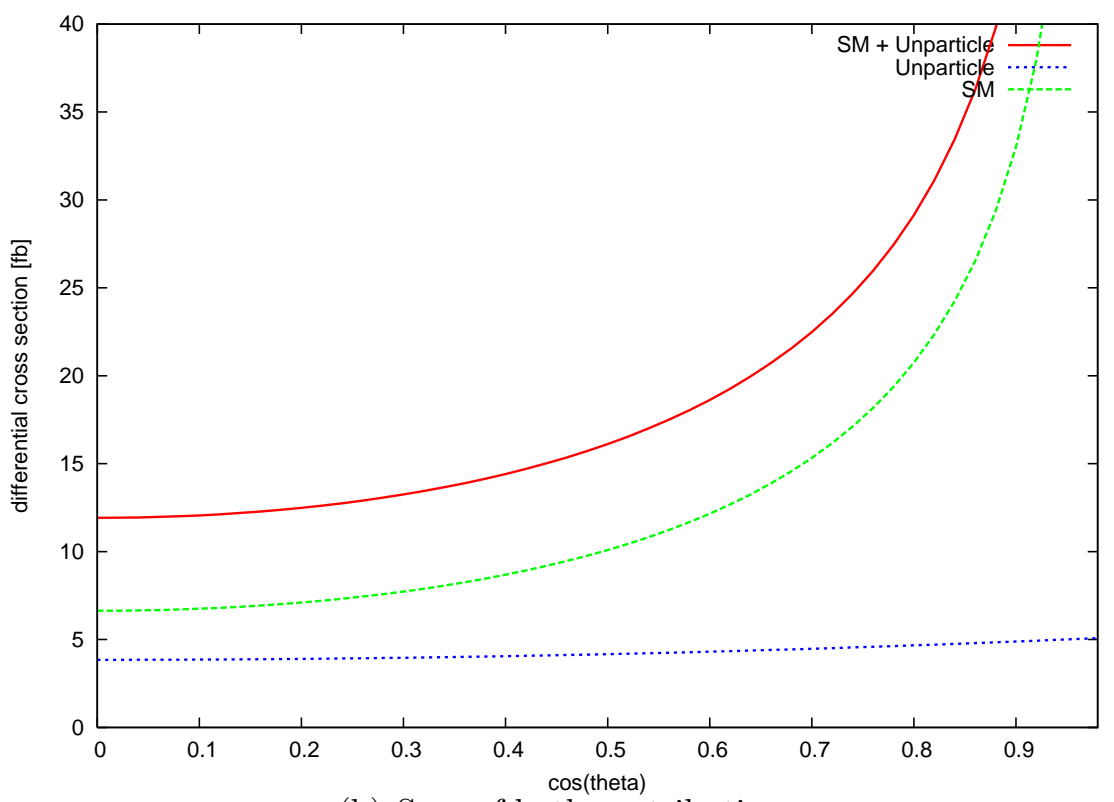

(b) Sum of both contributions

Figure 5: The angular distribution for the process, $\gamma \gamma \rightarrow \gamma \gamma$, including the unparticle in the intermediate state. In this figure, we have fixed the incident $e^{+} e^{-}$beam energy as $\sqrt{s}=500$ $\mathrm{GeV}$. The top figure shows each contribution for $\left(\lambda_{1}, \lambda_{2}\right)=( \pm \pm)$ and $( \pm \mp)$. The bottom figure shows the sum of both contributions. 


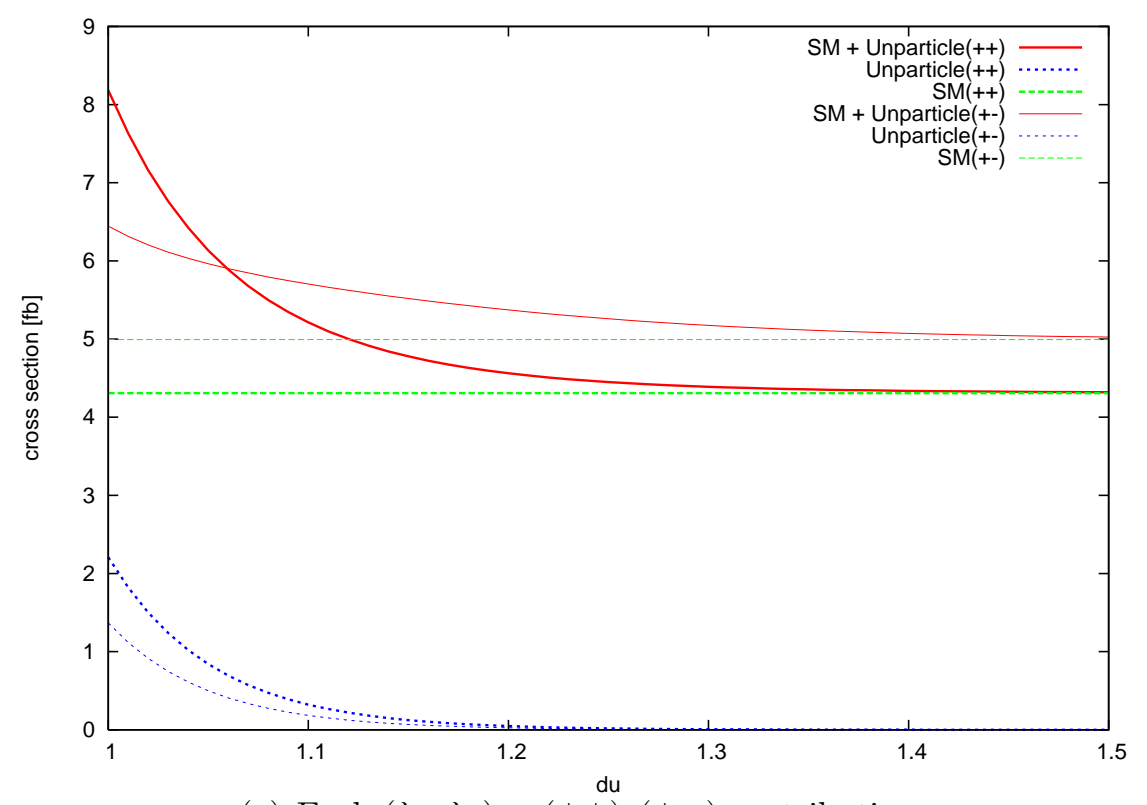

(a) Each $\left(\lambda_{1}, \lambda_{2}\right)=( \pm \pm)^{d u},( \pm \mp)$ contribution

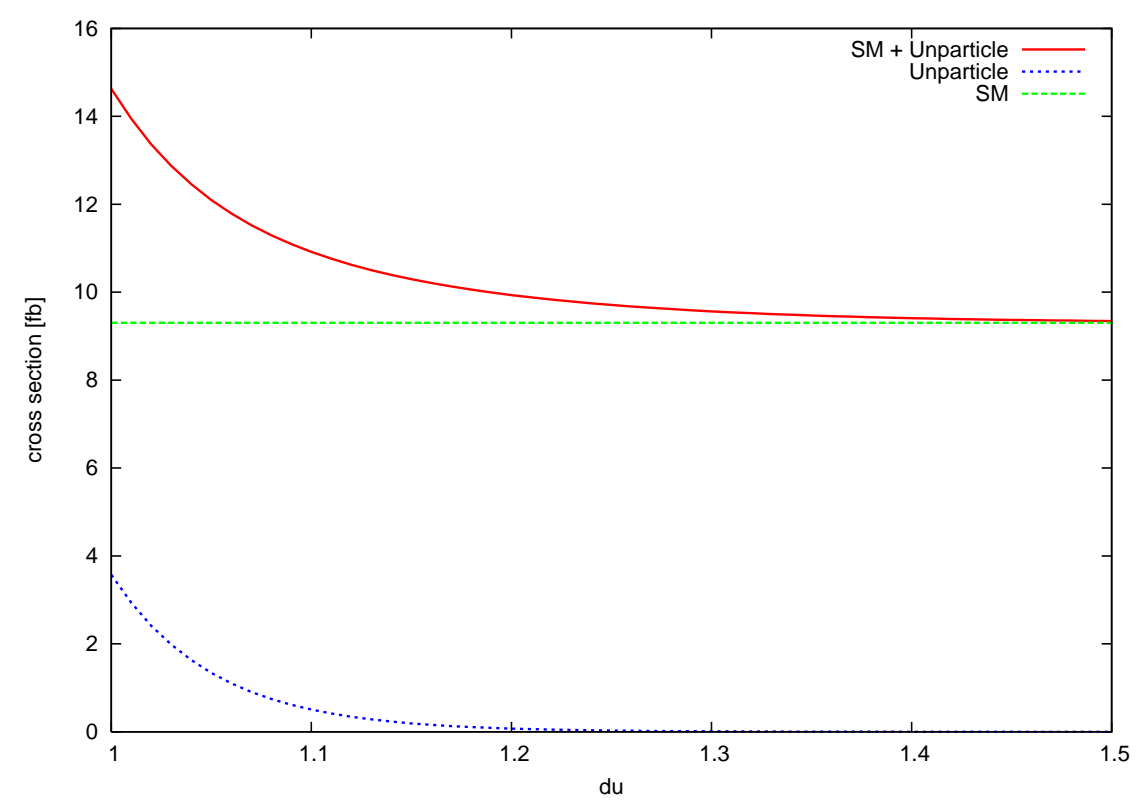

(b) Sum of both contributions

Figure 6: The total cross section of the $\gamma \gamma \rightarrow \gamma \gamma$ process for the Standard Model contribution, pure unparticle contribution and the combined result as a function of the scaling dimension, $d_{\mathcal{U}}$. We have fixed the incident $e^{+} e^{-}$beam energy as $\sqrt{s}=500 \mathrm{GeV}$ and $\Lambda=5$ $\mathrm{TeV}$. A cut for the scattering angle is taken to be $30^{\circ}<\theta<150^{\circ}$. The top figure shows each contribution for $\left(\lambda_{1}, \lambda_{2}\right)=( \pm \pm)$ and $( \pm \mp)$. The bottom figure shows the sum of both contributions. 


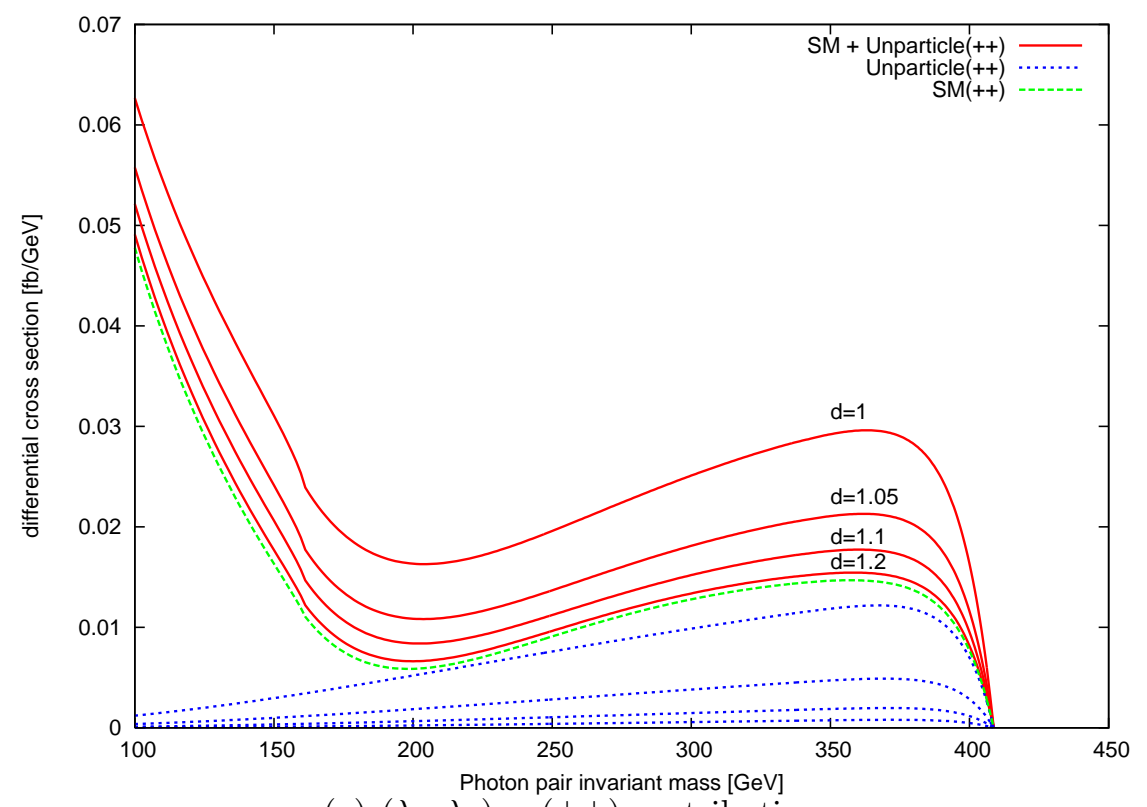

(a) $\left(\lambda_{1}, \lambda_{2}\right)=( \pm \pm)$ contributions

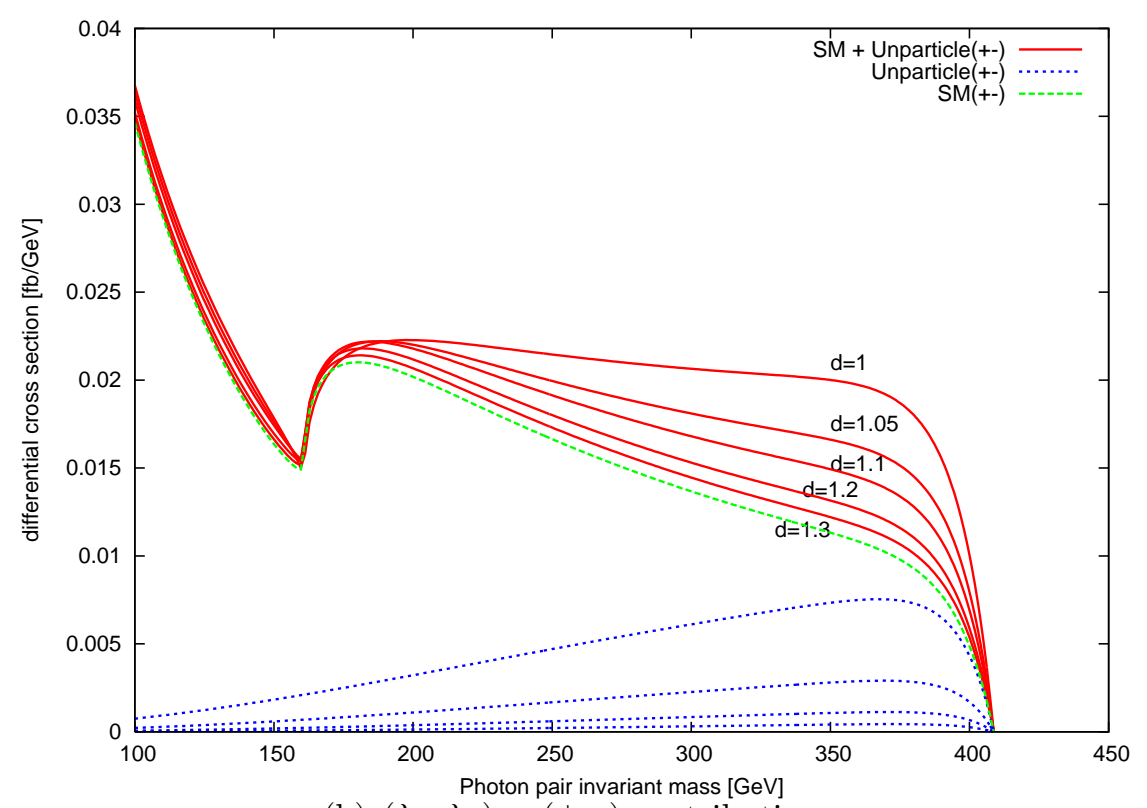

(b) $\left(\lambda_{1}, \lambda_{2}\right)=( \pm \mp)$ contributions

Figure 7: The differential cross section $d \sigma / d M_{\gamma \gamma}$ for the process $\gamma \gamma \rightarrow \gamma \gamma\left(30^{\circ}<\theta<150^{\circ}\right)$ as a function of a final state photon invariant mass $M_{\gamma \gamma}$. The energy is fixed to $\sqrt{s}=500$ $\mathrm{GeV}$ and $\Lambda=5 \mathrm{TeV}$. Here we show each contribution from different incident photon beam helicity combination separately The top figure is for $\left(\lambda_{1}, \lambda_{2}\right)=( \pm \pm)$ and the bottom is for $( \pm \mp)$. Each curve corresponds to $d_{\mathcal{U}}=1,1.05,1.1,1.2,1.3$. 


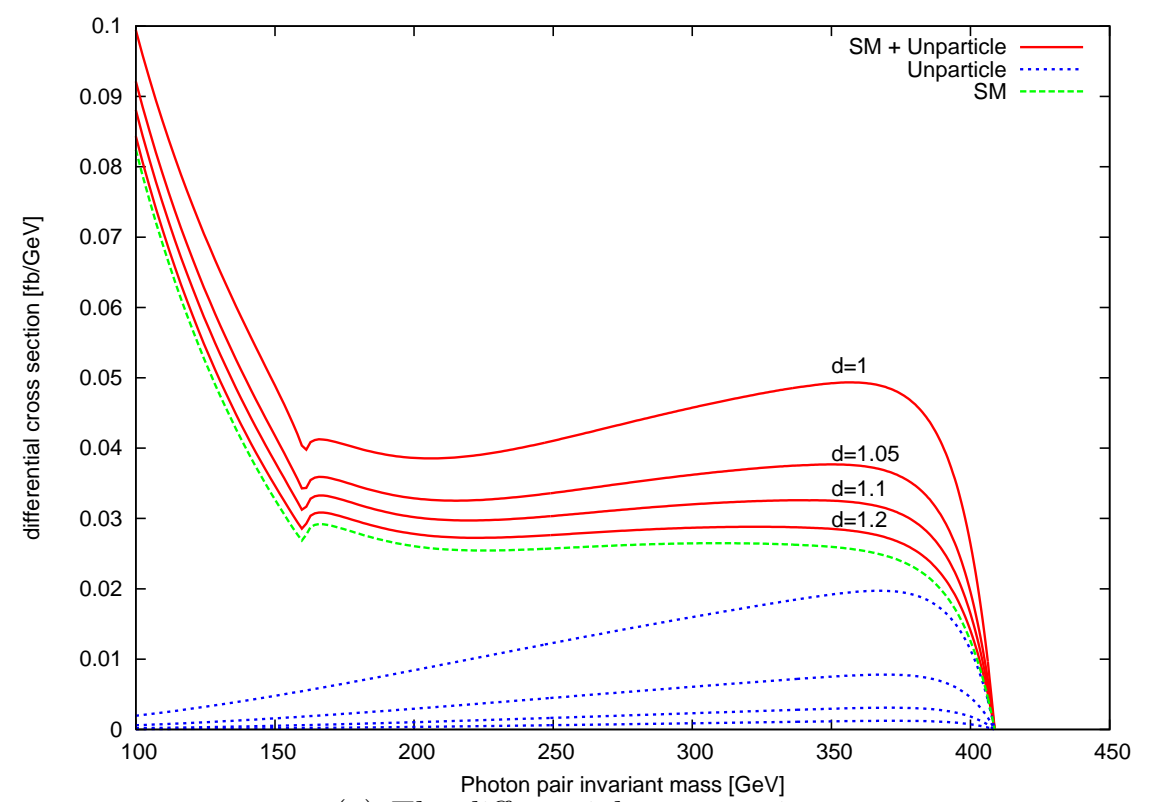

(a) The differential cross section

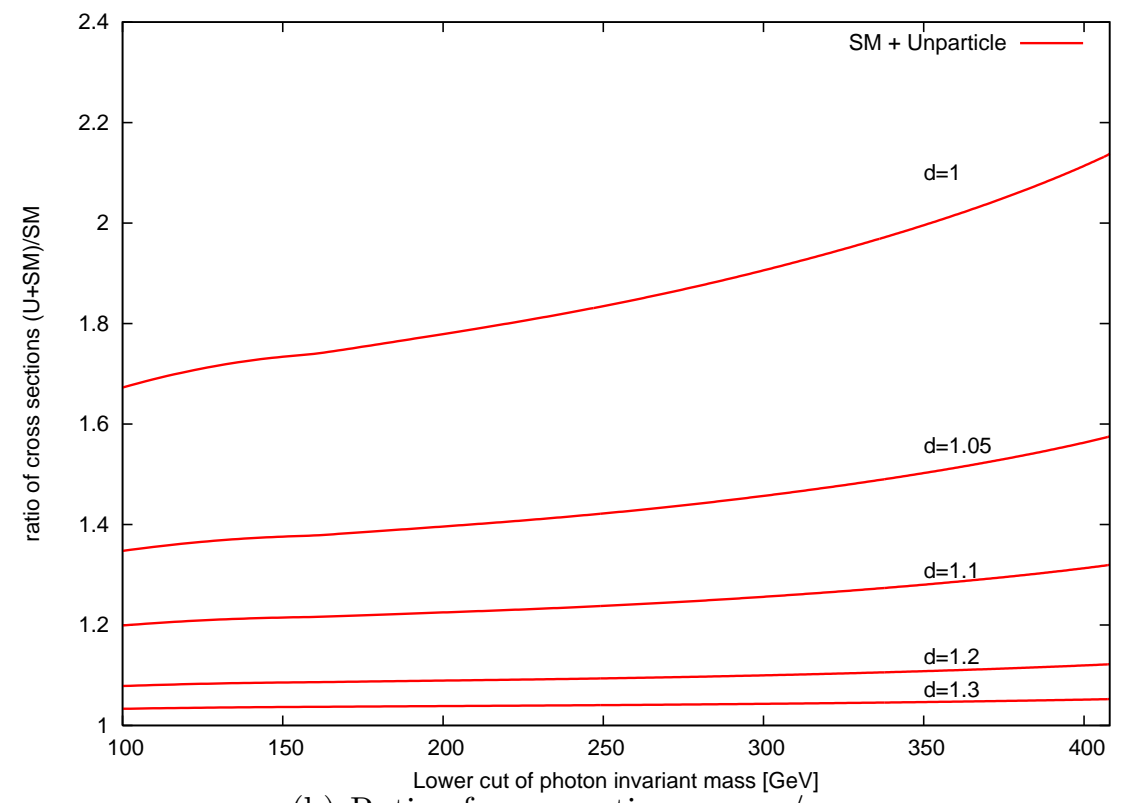

(b) Ratio of cross section $\sigma_{\mathcal{U}+\mathrm{SM}} / \sigma_{\mathrm{SM}}$

Figure 8: The total cross section for the case of unpolarized beams. Fig. 8(a) shows the same figure as Fig. 7 but the initial helicities are summed up to show the unpolarized cross sections. Fig. 8(b) shows the ratio of the signal cross section to the SM cross section $\left(\sigma_{\mathcal{U}+\mathrm{SM}} / \sigma_{\mathrm{SM}}\right)$ as a function of a lower energy cut on the final state photon invariant mass $M_{\gamma \gamma}^{\text {cut }}$. 


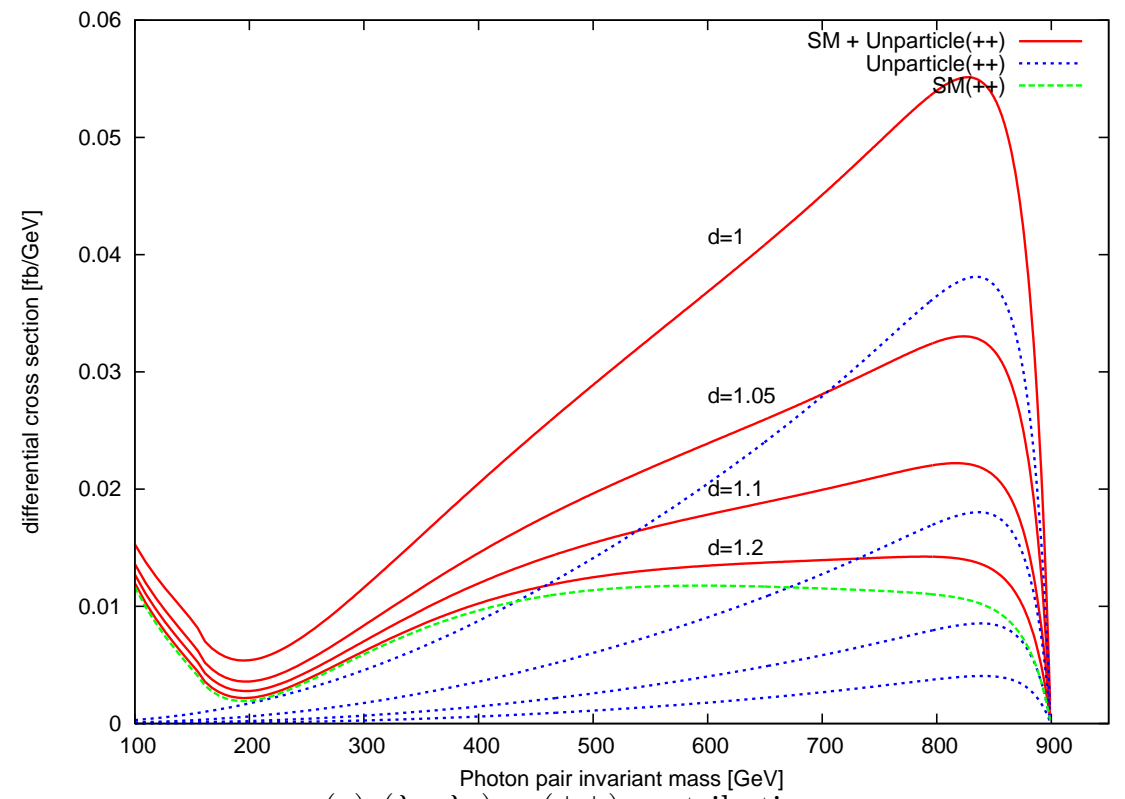

(a) $\left(\lambda_{1}, \lambda_{2}\right)=( \pm \pm)$ contributions

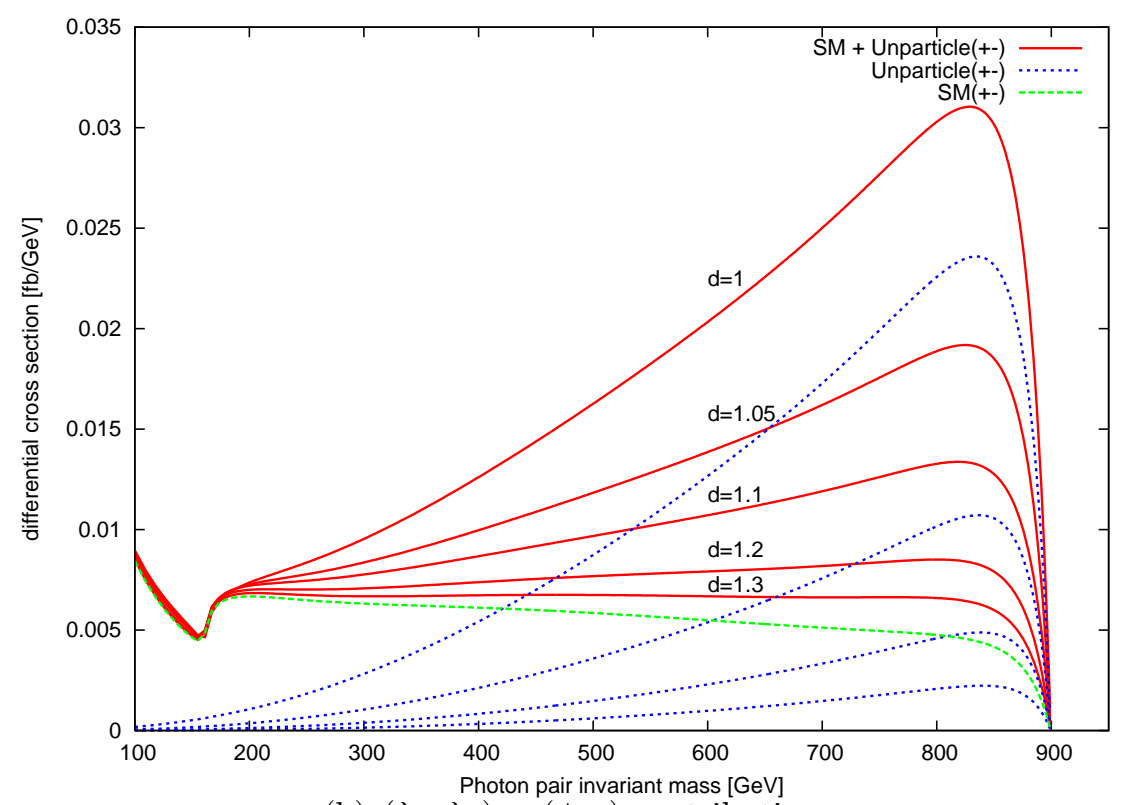

(b) $\left(\lambda_{1}, \lambda_{2}\right)=( \pm \mp)$ contributions

Figure 9: The same figure as Fig. 7, but for the case of $\sqrt{s}=1 \mathrm{TeV}$. 


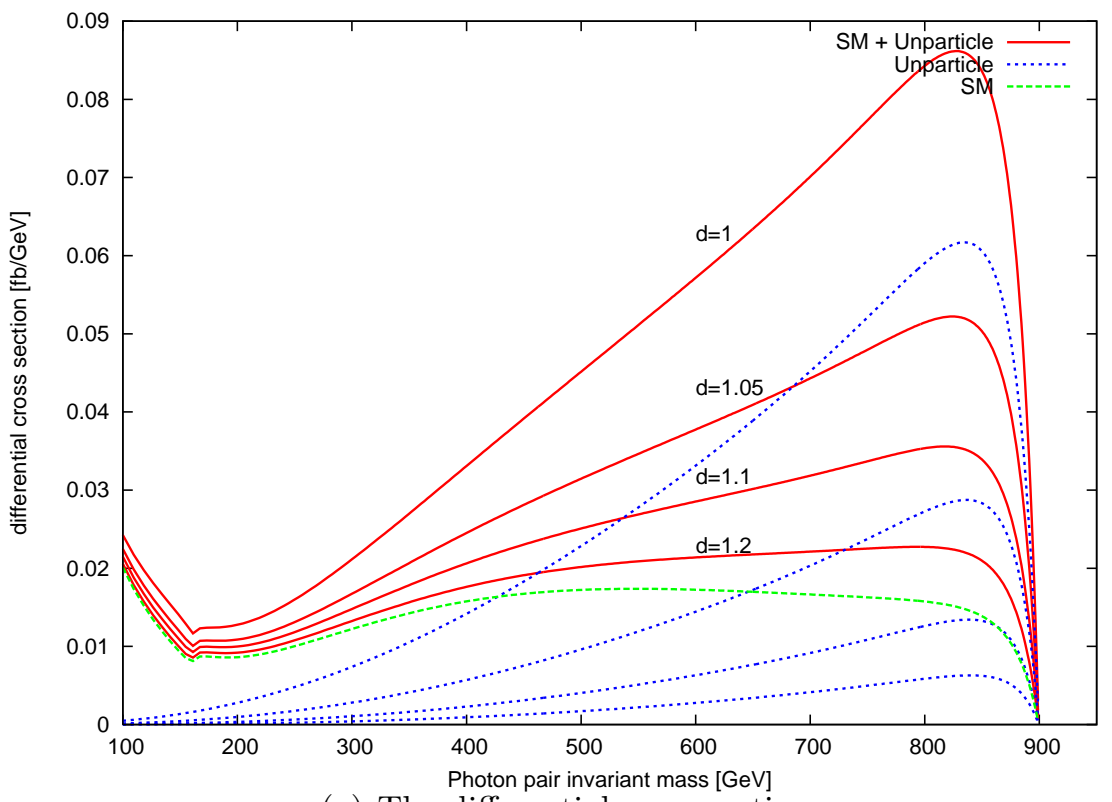

(a) The differential cross section

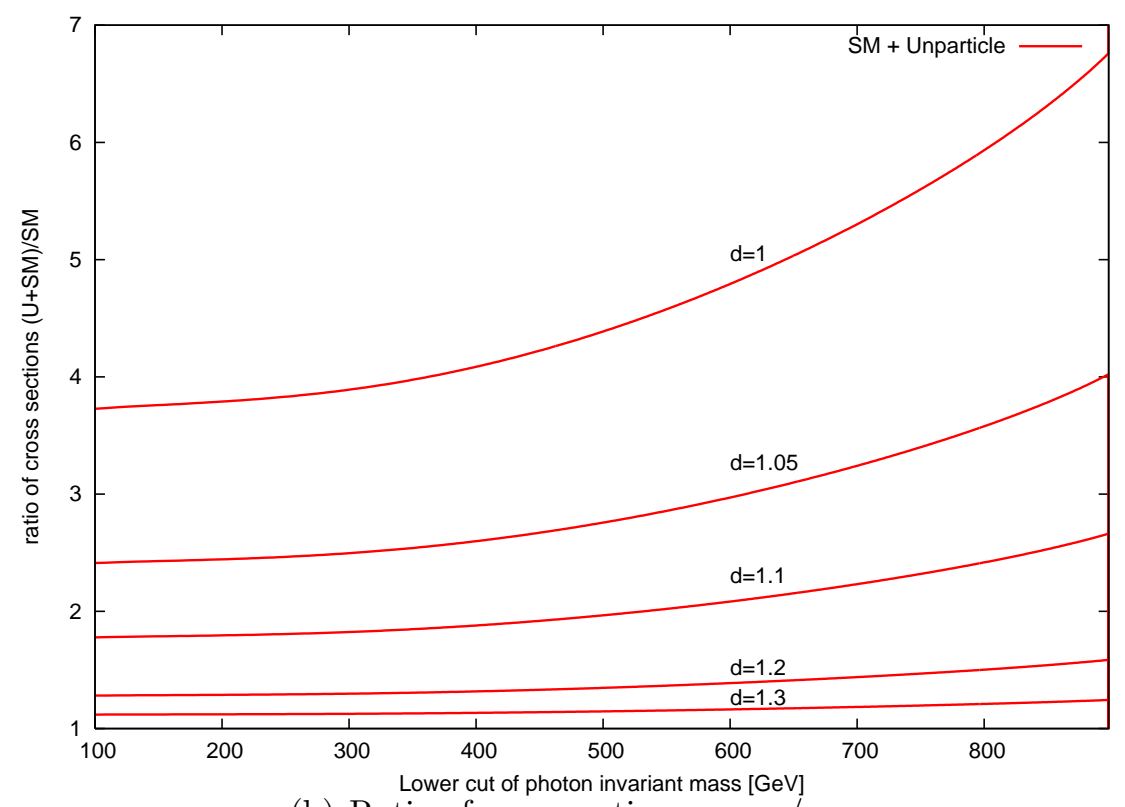

(b) Ratio of cross section $\sigma_{\mathcal{U}+\mathrm{SM}} / \sigma_{\mathrm{SM}}$

Figure 10: The same figure as Fig. 8 , but for the case of $\sqrt{s}=1 \mathrm{TeV}$. 


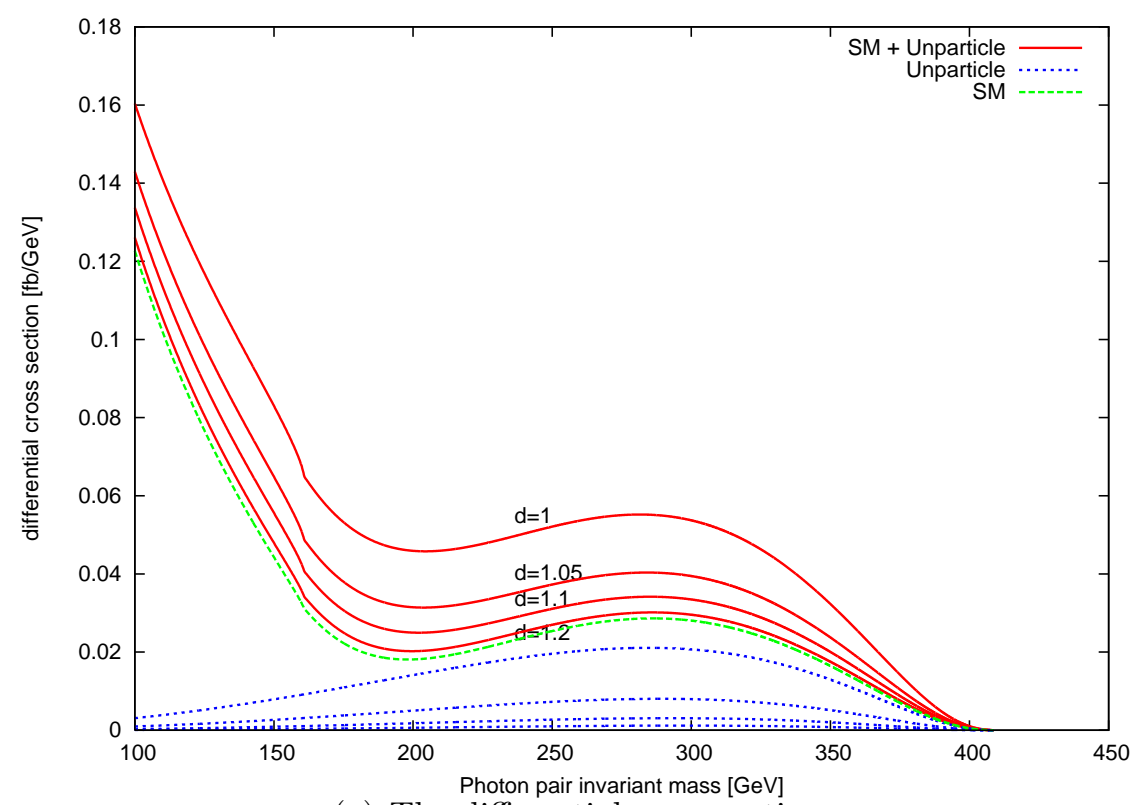

(a) The differential cross section

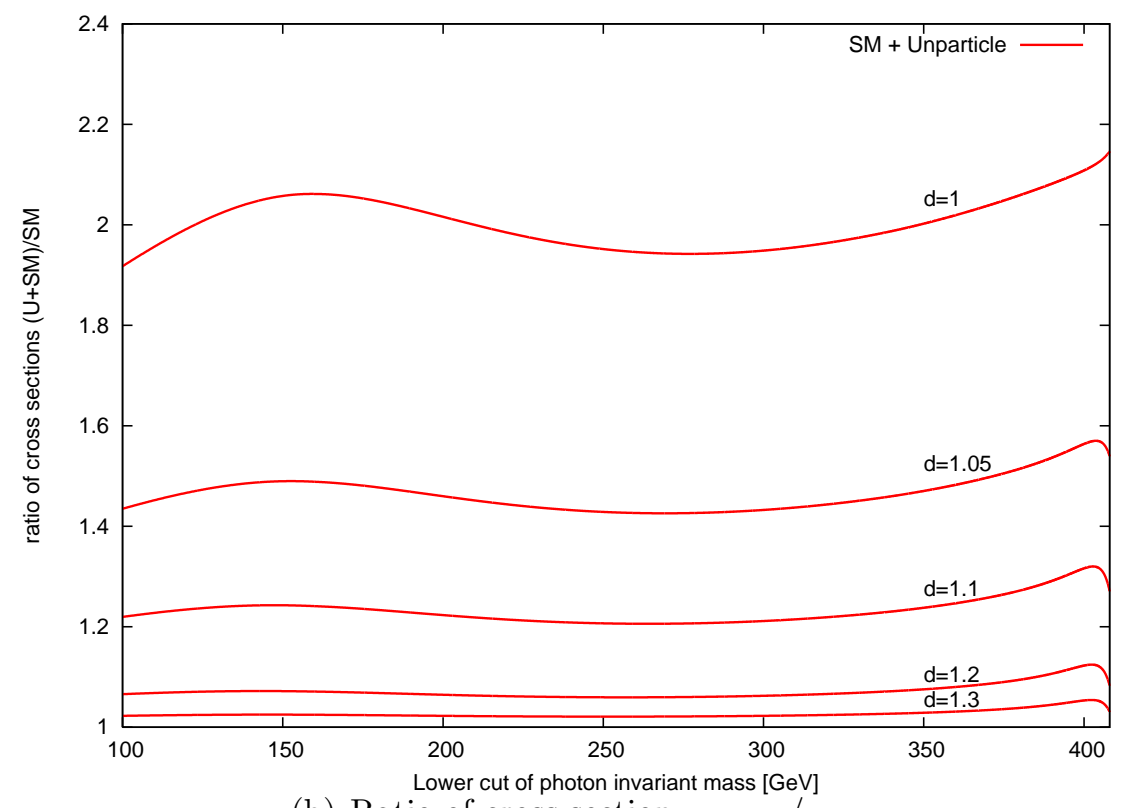

(b) Ratio of cross section $\sigma_{\mathcal{U}+\mathrm{SM}} / \sigma_{\mathrm{SM}}$

Figure 11: The same figure as Fig. 8 but the beam polarizations are chosen as $\left(P_{e}, P_{\ell}, P_{e}^{\prime}, P_{\ell}^{\prime}\right)=( \pm \pm \pm \pm)$. 


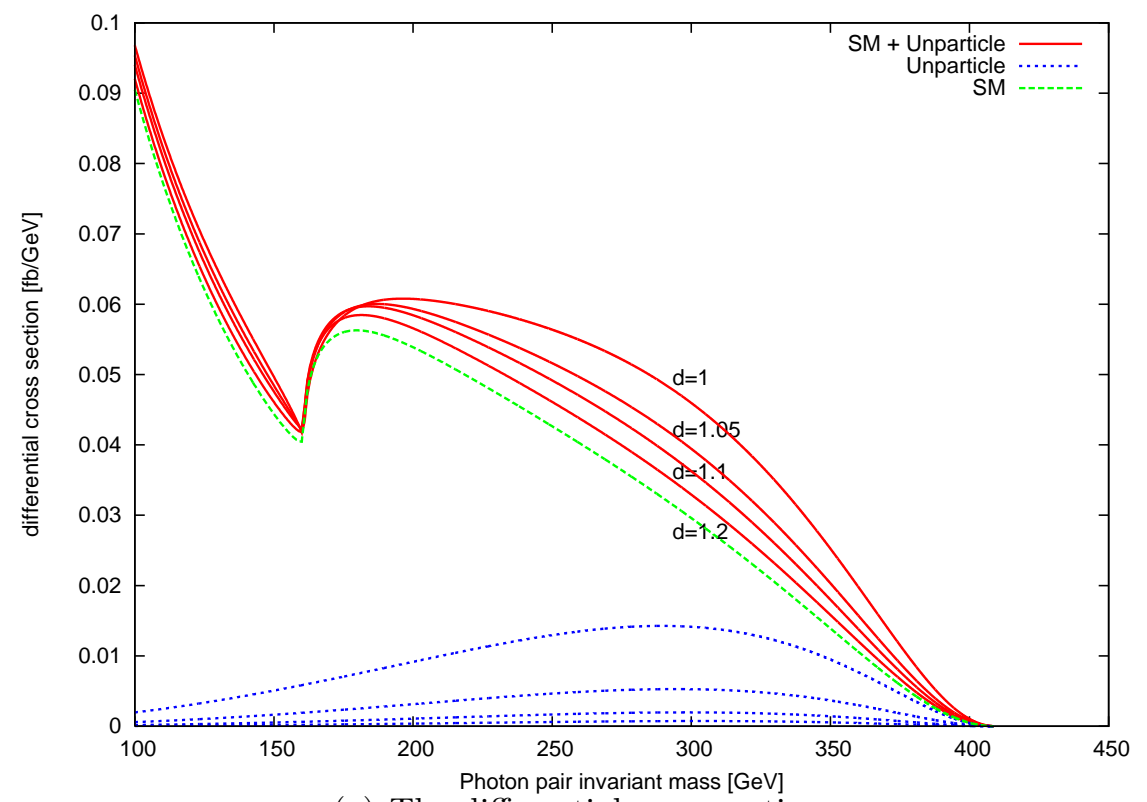

(a) The differential cross section

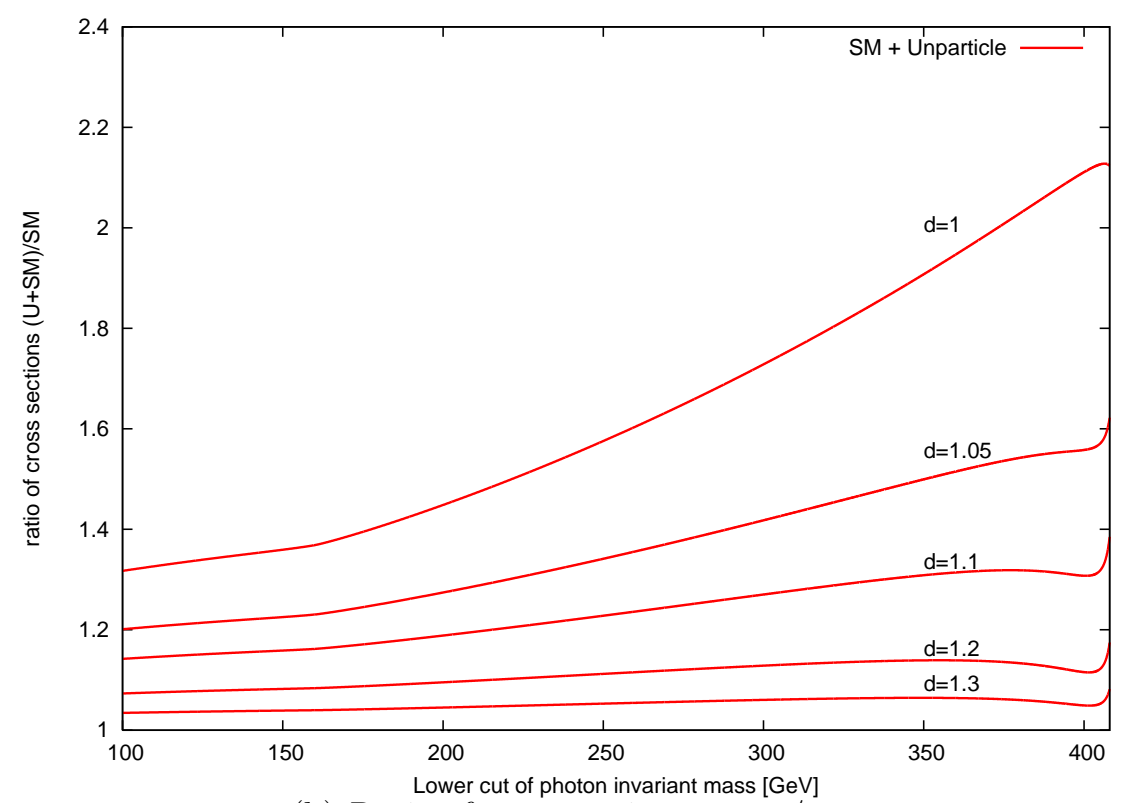

(b) Ratio of cross section $\sigma_{\mathcal{U}+\mathrm{SM}} / \sigma_{\mathrm{SM}}$

Figure 12: The same figure as Fig. 8 but the beam polarizations are chosen as $\left(P_{e}, P_{\ell}, P_{e}^{\prime}, P_{\ell}^{\prime}\right)=( \pm \pm \mp \mp)$. 


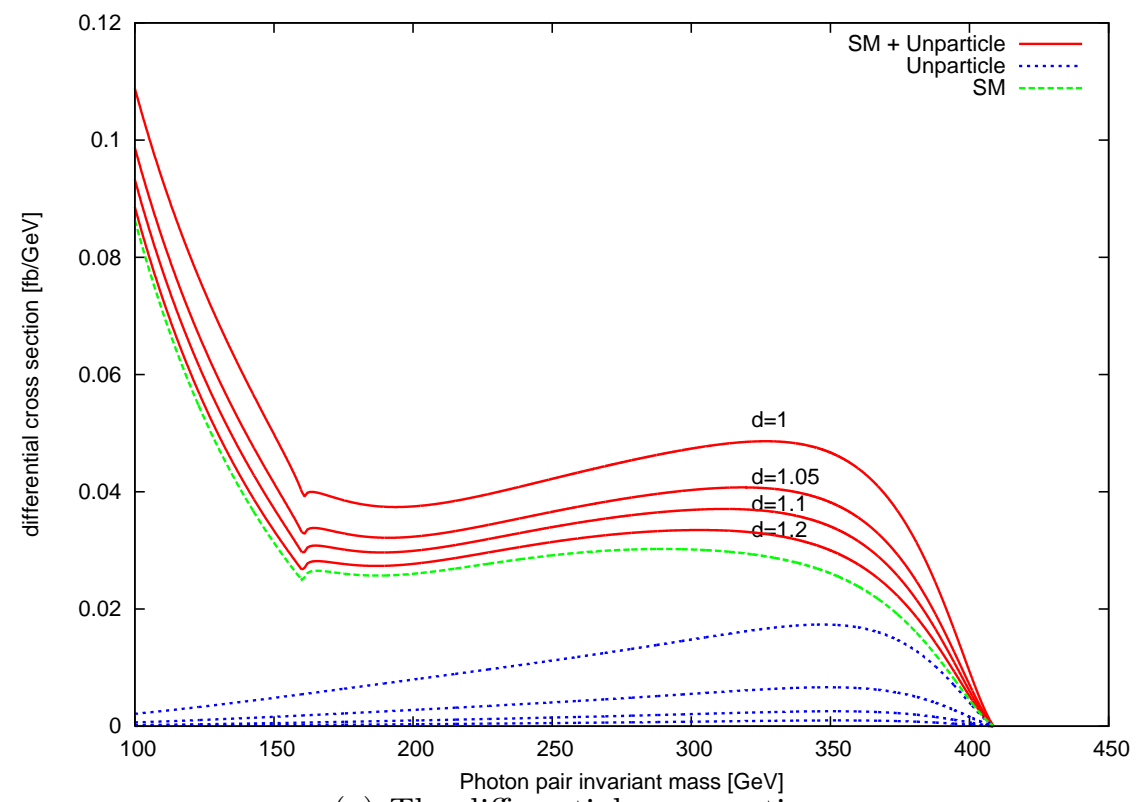

(a) The differential cross section

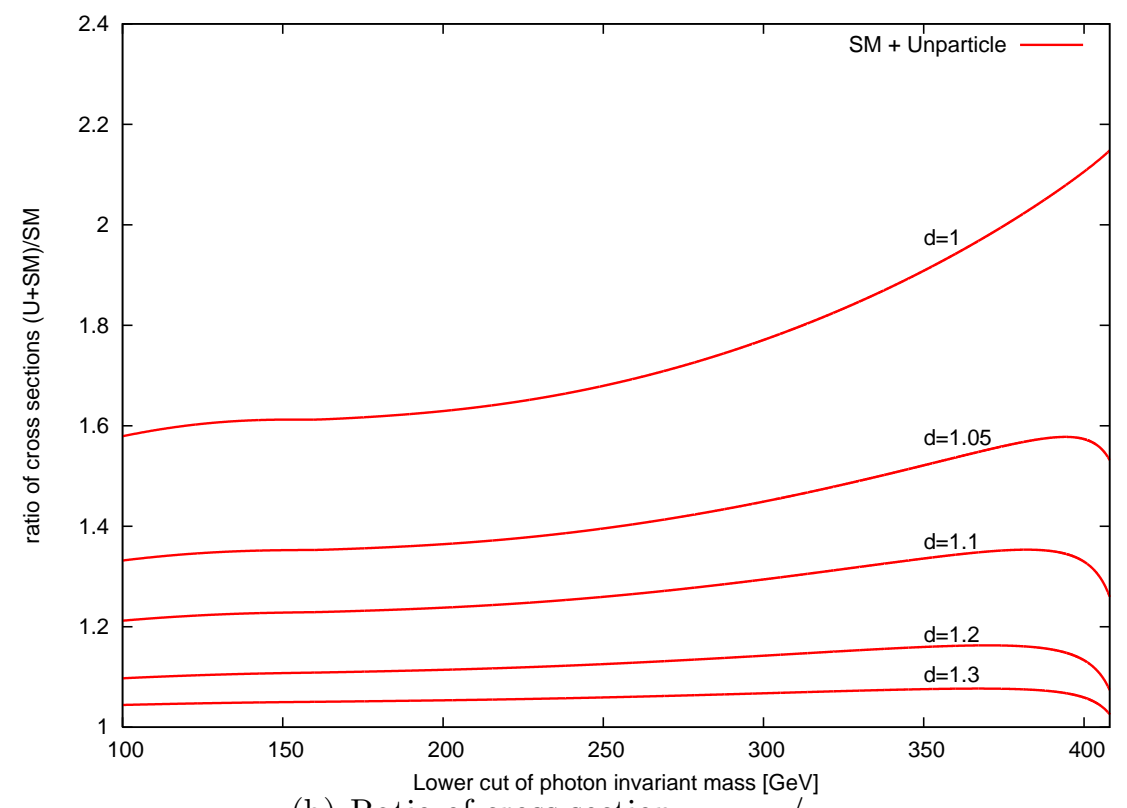

(b) Ratio of cross section $\sigma_{\mathcal{U}+\mathrm{SM}} / \sigma_{\mathrm{SM}}$

Figure 13: The same figure as Fig. 8 but the beam polarizations are chosen as $\left(P_{e}, P_{\ell}, P_{e}^{\prime}, P_{\ell}^{\prime}\right)=( \pm \pm \mp \pm)$ or $(\mp \pm \pm \pm)$. 


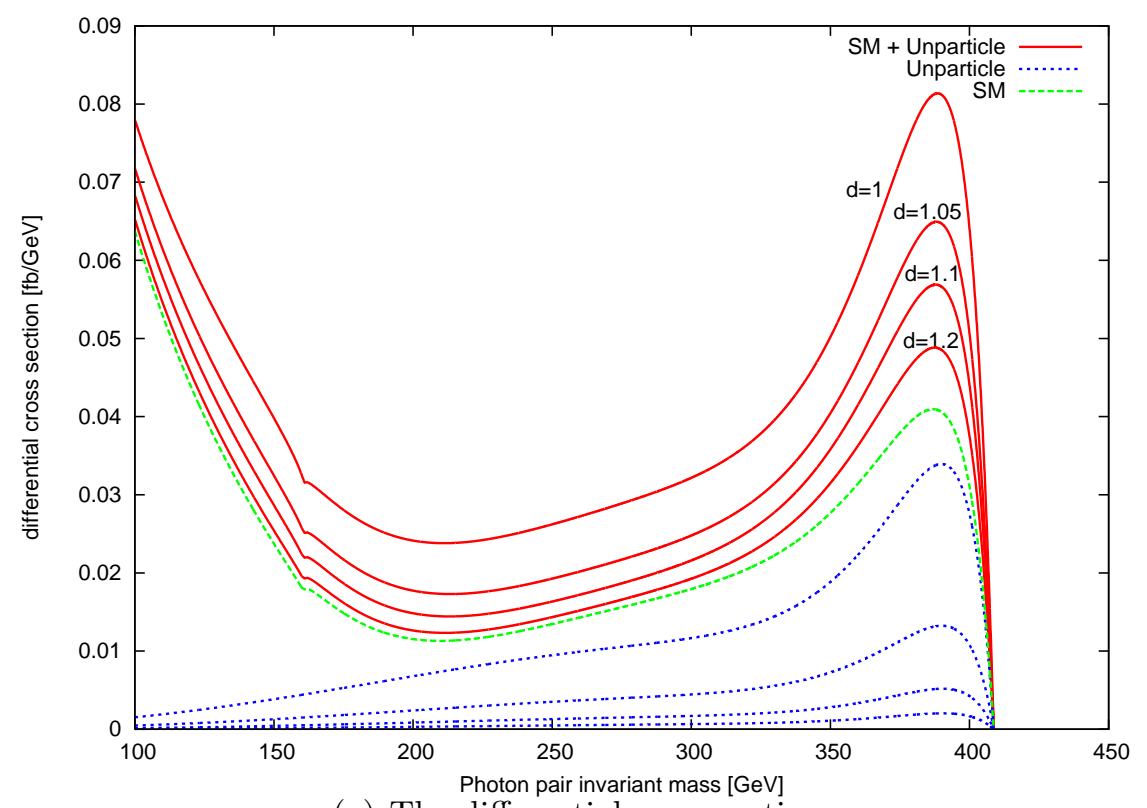

(a) The differential cross section

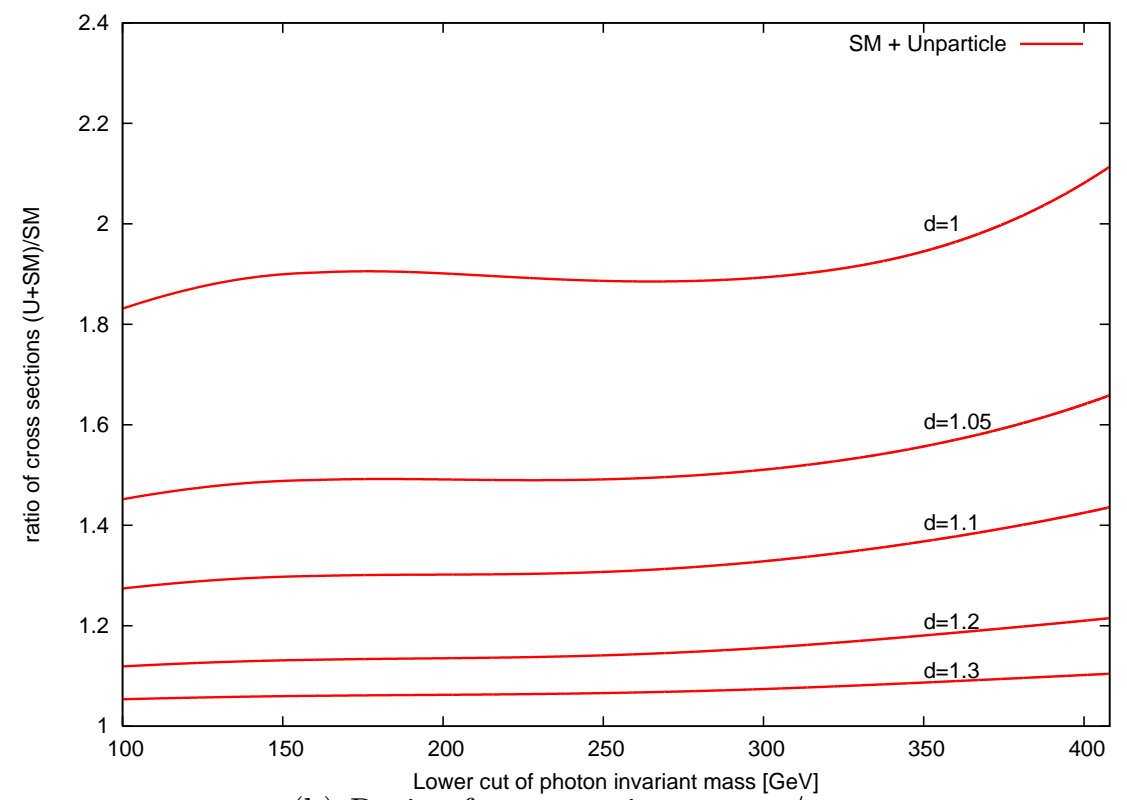

(b) Ratio of cross section $\sigma_{\mathcal{U}+\mathrm{SM}} / \sigma_{\mathrm{SM}}$

Figure 14: The same figure as Fig. 8 but the beam polarizations are chosen as $\left(P_{e}, P_{\ell}, P_{e}^{\prime}, P_{\ell}^{\prime}\right)=( \pm \mp \mp \pm)$. 


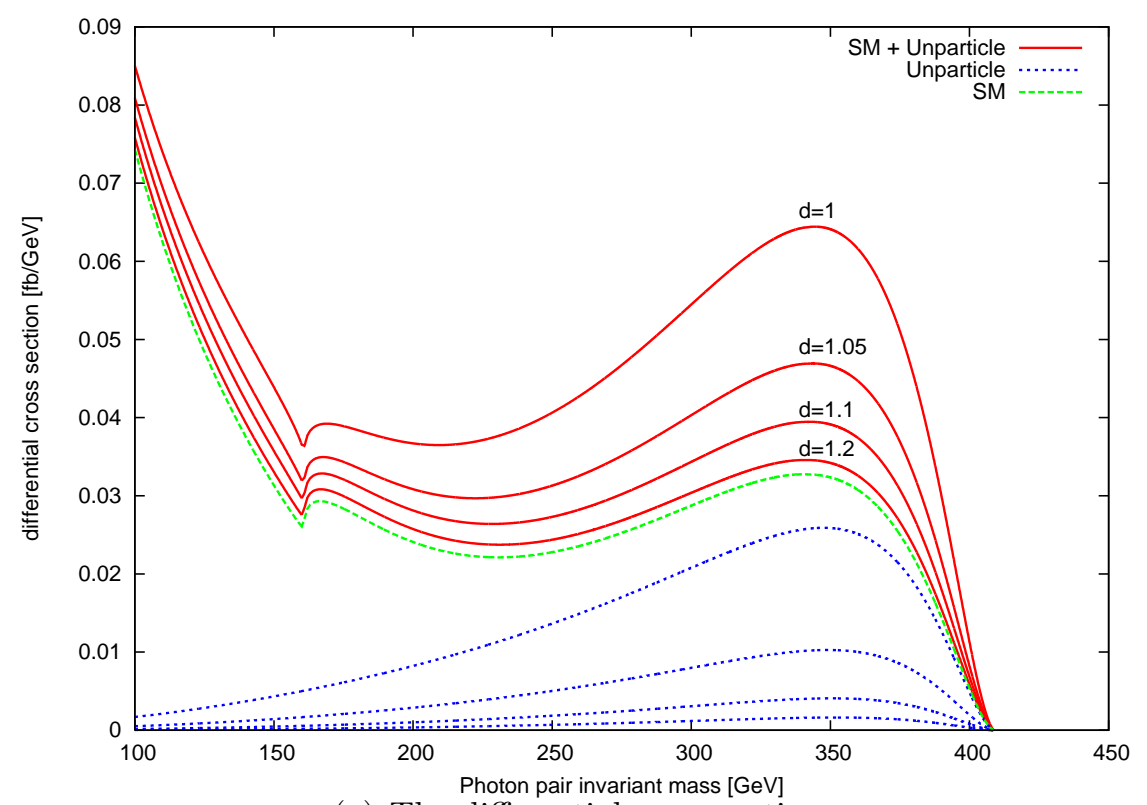

(a) The differential cross section

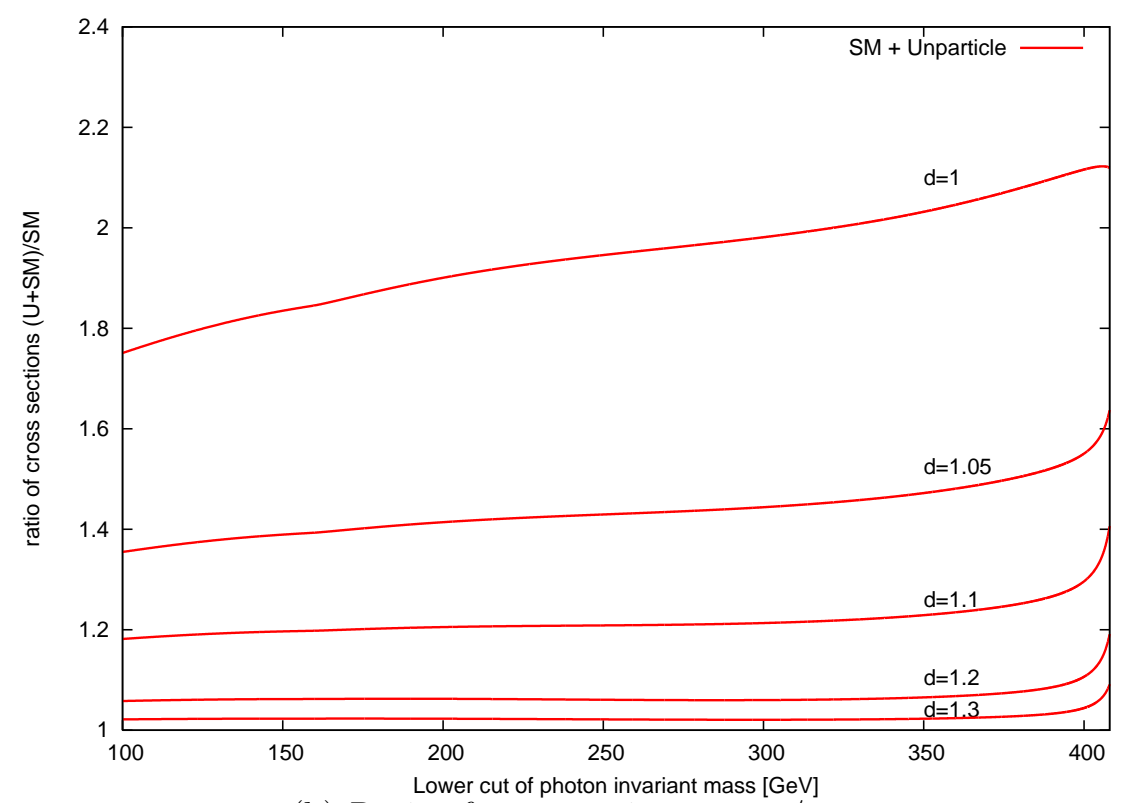

(b) Ratio of cross section $\sigma_{\mathcal{U}+\mathrm{SM}} / \sigma_{\mathrm{SM}}$

Figure 15: The same figure as Fig. 8 but the beam polarizations are chosen as $\left(P_{e}, P_{\ell}, P_{e}^{\prime}, P_{\ell}^{\prime}\right)=( \pm \pm \pm \mp)$ or $( \pm \mp \pm \pm)$. 


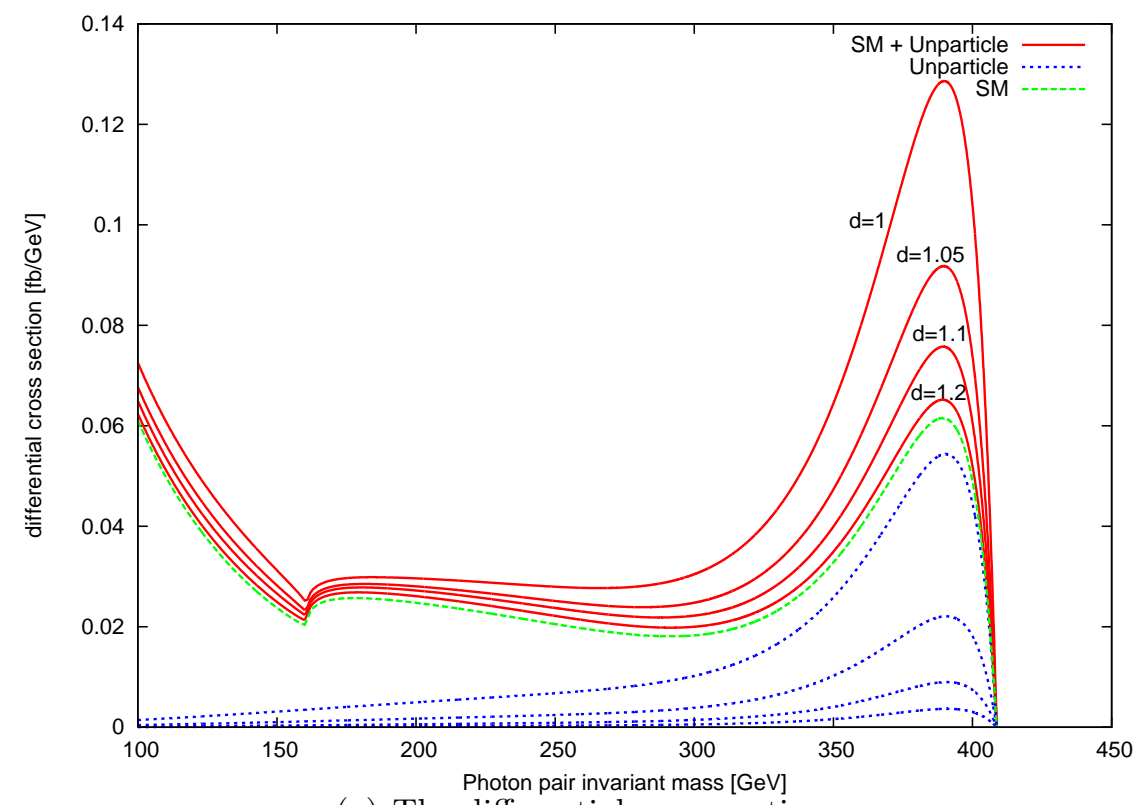

(a) The differential cross section

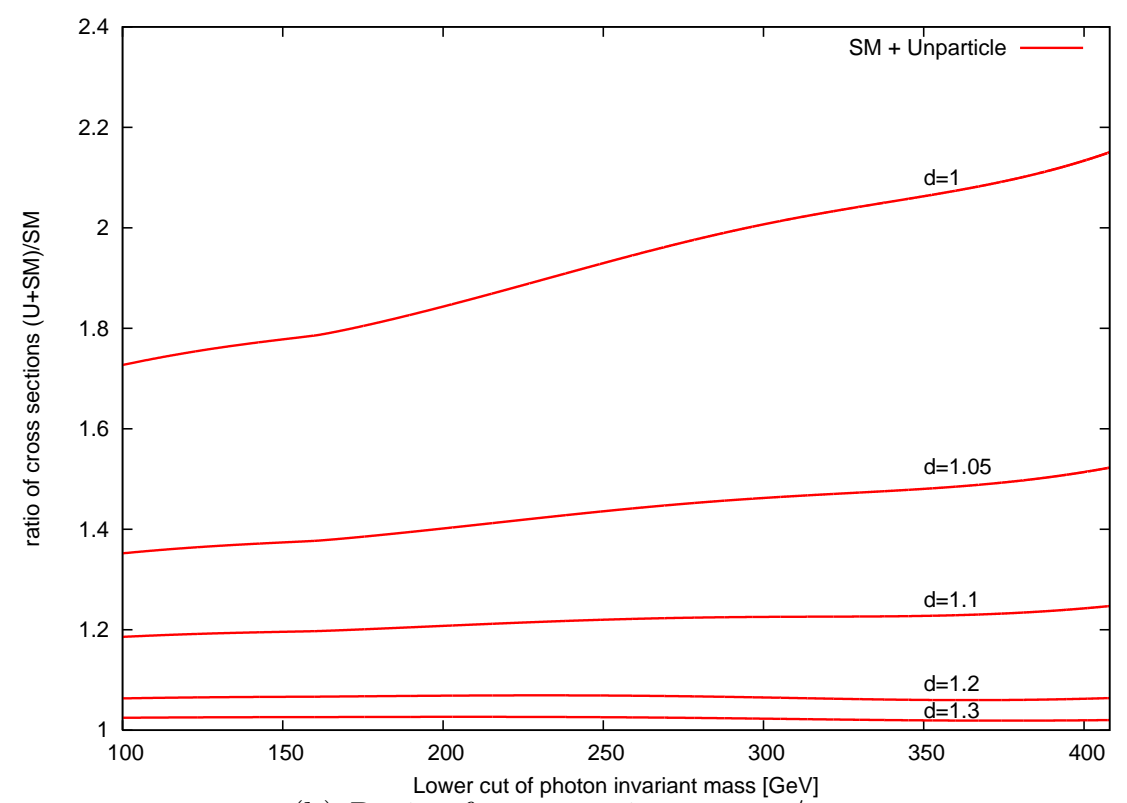

(b) Ratio of cross section $\sigma_{\mathcal{U}+\mathrm{SM}} / \sigma_{\mathrm{SM}}$

Figure 16: The same figure as Fig. 8 but the beam polarizations are chosen as $\left(P_{e}, P_{\ell}, P_{e}^{\prime}, P_{\ell}^{\prime}\right)=( \pm \mp \pm \mp)$. 